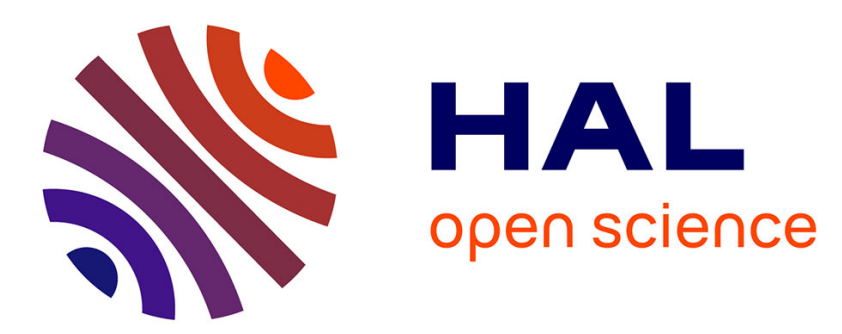

\title{
Fast and accurate pricing of barrier options under Lévy processes
}

Oleg Kudryavtsev, Sergei Levendorskii

\section{To cite this version:}

Oleg Kudryavtsev, Sergei Levendorskii. Fast and accurate pricing of barrier options under Lévy processes. [Research Report] RR-6670, INRIA. 2008, pp.40. inria-00332224

\section{HAL Id: inria-00332224 \\ https://hal.inria.fr/inria-00332224}

Submitted on 20 Oct 2008

HAL is a multi-disciplinary open access archive for the deposit and dissemination of scientific research documents, whether they are published or not. The documents may come from teaching and research institutions in France or abroad, or from public or private research centers.
L'archive ouverte pluridisciplinaire HAL, est destinée au dépôt et à la diffusion de documents scientifiques de niveau recherche, publiés ou non, émanant des établissements d'enseignement et de recherche français ou étrangers, des laboratoires publics ou privés. 
INSTITUT NATIONAL DE RECHERCHE EN INFORMATIQUE ET EN AUTOMATIQUE

Fast and accurate pricing of barrier options under Lévy processes

Oleg Kudryavtsev — Sergei Levendorskii

$\mathbf{N}^{\circ} 6670$

Octobre 2008

Thème NUM

apport

derecherche 



\title{
Fast and accurate pricing of barrier options under Lévy processes
}

\author{
Oleg Kudryavtsev*, Sergei Levendorski讯 \\ Thème NUM - Systèmes numériques \\ Équipe-Projet Mathfi \\ Rapport de recherche $n^{\circ} 6670$ - Octobre 2008 - 37 pages
}

\begin{abstract}
We suggest two new fast and accurate methods, Fast Wiener-Hopf method (FWH-method) and Iterative Wiener-Hopf method (IWH-method), for pricing barrier options for a wide class of Lévy processes. Both methods use the Wiener-Hopf factorization and Fast Fourier Transform algorithm. Using an accurate albeit relatively slow finite-difference algorithm developed in Levendorskii et al (2006) (FDS-method), we demonstrate the accuracy and fast convergence of the two methods for processes of finite variation. We explain that the convergence of the methods must be better for processes of infinite variation, and, as a certain supporting evidence, demonstrate with numerical examples that the results obtained by two methods are in extremely good agreement. Finally, we use FDS, FWH and IWH-methods to demonstrate that Cont and Volchkova method (CV-method), which is based on the approximation of small jumps by an additional diffusion, may lead to sizable relative errors, especially near the barrier and strike. The reason is that $\mathrm{CV}$-method presumes that the option price is of class $C^{2}$ up to the barrier, whereas for processes without Gaussian component, it is typically not of class $C^{1}$ at the barrier.
\end{abstract}

Key-words: Lévy processes, barrier options, Wiener-Hopf factorization, numerical methods

\footnotetext{
* Department of Informatics, Russian Customs Academy Rostov Branch, Budennovskiy 20, Rostov-on-Don, 344004, Russia - E-mail: koe@donrta.ru,okudr@mail.ru

$\dagger$ Department of Economics, The University of Texas at Austin, 1 University Station C3100, Austin, TX 78712-0301 - E-mail: leven@eco.utexas.edu
}

Centre de recherche INRIA Paris - Rocquencourt 


\section{Méthode rapide et précise pour l'évaluation d'options à barrières dans les modèles de Lévy}

Résumé : On présente deux nouvelles méthodes performantes, Fast WienerHopf method (FWH-method) et Iterative Wiener-Hopf method (IWH-method), pour évaluer des options barrières pour une grande classe de processus de Lévy. Ces deux méthodes utilisent la factorisation de Wiener-Hopf et l'algorithme de Transformée de Fourier rapide (FFT). En utilisant un algorithme de différences finies précis mais relativement lent développé par Levendorskii et al (2006) (FDS-method), on démontre la précision et la convergence rapide des deux méthodes pour des processus à variation finie. On explique que la convergence des méthodes doit être meilleure pour des processus à variation infinie et on montre sur des exemples numériques la cohérence des résultats obtenus par les deux méthodes. Finalement on utilise les méthodes FDS, FWH and IWHmethods pour montrer que la méthode de Cont and Volchkova (CV-method), qui est basée sur l'approximation des petits sauts par une diffusion additionnelle peut entrainer des erreurs relativement importantes notamment près de la barrière et du strike. Cela s'explique par le fait que cette méthode présuppose que le prix de l'option est de classe $C^{2}$ jusqu'à la barrière, alors que pour des processus sans composante gaussienne, il n'est en général pas de classe $C^{1}$ à la barrière.

Mots-clés : Processus de Lévy, options à barrières, factorisation de WienerHopf, méthodes numériques 


\section{Introduction}

By now, there exist several large groups of relatively universal numerical methods for pricing of American and barrier options under exponential Lévy processes. The number of publications is huge, and, therefore, an exhaustive list is virtually impossible. We describe main groups of methods and several publications for each group, where the reader can find further references. Monte Carlo methods perform well for pricing of barrier options in jump-diffusion models when activity of jumps is finite because one can control the behavior of the process between the jump times, when the log-price follows a Browning bridge process (for details see Metwally and Atiya (2002), or Cont and Tankov (2004)). In the infinite activity case, the Monte Carlo methods are much less accurate and more time consuming. Evaluation of American option prices by Monte Carlo simulation faces additional difficulties: it involves the computation of conditional expectations - see, e.g., Longstaff and Schwartz (2001). An overview of Monte Carlo based methods for American option pricing can be found in Glasserman (2003) and Broadie and Detemple (2004). Generally, Monte Carlo methods consume much more time than other numerical methods.

Methods of the second large group start with the reduction to a boundary problem for the generalization of the Black-Scholes equation (backward Kolmogorov equation); in the case of American options, a free boundary problem arises. Boyarchenko and Levendorskii $(1999,2002 \mathrm{~b}, \mathrm{c})$ derived the equation for the price of a derivative security in the sense of the theory of generalized functions. Later, Cont and Volchkova (2005) (see also Cont and Tankov (2004)) derived the same equation under much more stringent conditions. The latter version is more popular because Cont and Volchkova (2005) call the equation partial integro-differential equation (PIDE) and use the viscosity solution technique, which are better known in probability than the language and technique of the theory of pseudo-differential operators (PDO) used by Boyarchenko and Levendorskii $(1999,2002 \mathrm{~b}, \mathrm{c})$. Notice, however, that the definition of a solution in the sense of generalized functions is standard in analysis for half a century, at least, and it is more natural for linear problems than the language of viscosity solutions invented to tackle much more difficult non-linear problems. Moreover, PDO technique based on the Fourier transfrom and the operator form of the Wiener-Hopf method is much more powerful than the technique based on the study of the kernel of the PIDE. This was the reason the theory of PDO was invented in the first place - see, e.g., Eskin (1973) and Hörmaner (1985). PDO technique allows one to study properties of solutions, which are crucial for development of accurate numerical methods. It is rather difficult to establish these properties working with the PIDE representation. In the result, in the majority of papers that use the PIDE language, construction of numerical methods presumes that solutions are more regular than they really are, and, in many cases, serious errors result as numerical examples below will demonstrate.

There are three main approaches for solving PIDE: multinomial trees, finite difference schemes and Galerkin methods. Amin (1993) constructed a family of 
Markov chain approximations of jump-diffusion models. Multinomial trees can be considered as special cases of explicit finite difference schemes. The main advantage of the method is simplicity of implementation; the drawbacks are inaccurate representation of the jumps and slow convergence. Galerkin methods are based on the variational formulation of PIDE. While implementation of finite difference methods requires only a moderate programming knowledge, Galerkin methods use specialized toolboxes. Finite difference schemes use less memory than Galerkin methods, since there is no overhead for managing grids, but a refinement of the grid is more difficult. A complicated wavelet Galerkin method for pricing American options under exponential Lévy processes is constructed in Matache et al. (2005). A general drawback of variational methods is that, for processes of finite variation, the convergence can be proved in the $H^{s}$-norm only, where $s<1 / 2$; hence, the convergence in $C$-norm is not guaranteed.

In a finite difference scheme, derivatives are replaced by finite differences. In the presence of jumps, one needs to discretize the integral term as well. Finite difference schemes were applied to pricing barrier options in Cont and Voltchkova (2005), and to pricing American options in Carr and Hirsa (2003), Hirsa and Madan (2003) and Levendorskil et al. (2005). Wang et al (2007) calculate prices of American options using the penalty method and a finite difference scheme. Construction of any finite difference scheme involves discretization in space and time, truncation of large jumps and approximation of small jumps. Truncation of large jumps is necessary because an infinite sum cannot be calculated; approximation of small jumps is needed when Lévy measure diverges at zero. The result is a linear system that needs to be solved at each time step, starting from payoff function. In the general case, solution of the system on each time step by a linear solver requires $O\left(m^{2}\right)$ operations $(m$ is a number of space points), which is too time consuming. In Carr and Hirsa (2003), Hirsa and Madan (2003), and Cont and Voltchkova (2005) the integral part is computed using the solution from the previous time step, while the differential term is treated implicitly. This leads to the explicit-implicit scheme, with tridiagonal system which can be solved in $O(m \ln m)$ operations. Levendorskii et al. (2005) use the implicit scheme and the iteration method at each time step. The methods in Carr and Hirsa (2003), Hirsa and Madan (2003) and Levendorskii et al. (2006) are applicable to processes of infinite activity and finite variation; the part of the infinitesimal generator corresponding to small jumps is approximated by a differential operator of first order (additional drift component). Cont and Volchkova (2005) use an approximation by a differential operator of second order (additional diffusion component). The discretization scheme for PIDE in Albanese and Kuznetsov (2003) is applicable to models for which the spectrum of the infinitesimal generator can be computed in analytically closed form.

Comparing the groups of methods described above, one is tempted to conclude that finite difference methods should be preferred as rather simple, fairly fast and reasonably accurate methods. In particular, a popular Cont and Volchkova (2005) method (CV-method) looks attractive due to its simplicity. However, as it was explained in Levendorskii (2004b) (see also Section 2), finite difference methods based on approximation of small jumps by an additional diffusion component must lead to serious errors if the underlying process itself has no diffusion component or small diffusion component; the numerical examples in the main body of this paper demonstrate that the error can be quite sizable. 
Note that as empirical studies of financial markets show, typically, there is no diffusion component in stock dynamics (see, e.g., Carr et al. (2002)), therefore this issue is important. The last group of methods described below is free of errors of this sort because no crude approximation of small jumps is involved.

The fourth group of methods use the Wiener-Hopf factorization method, which is more adequate than other methods for study of boundary problems for pseudodifferential equation in general, and PIDE in particular. There are several versions of the Wiener-Hopf factorization method. In a special case of double exponential jump-diffusion model, Lipton (2002), Kou and Wang (2003) and Sepp (2004) derive explicit formulas for the Laplace transforms of barrier, double barrier and lookback option prices. However, the inversion of the Laplace transform is well-known ill-posed problem; quite often, it is difficult to calculate accurately prices of much simpler European options using the Fourier transform - see Chapter 12 in Boyarchenko and Levendorkii (2002b) and N.Boyarchenko and Levendorskii (2007). In Lipton (2002), Kou and Wang (2003), Sepp (2004), and in a number of other papers, e.g., Avram et al. (2002) and Asmussen et al. (2004), prices are derived from the distribution of the first passage time; the distribution is calculated using the Wiener-Hopf factorization method in the form used in probability. See also Kyprianou and Pistorius (2003), Alili and Kyprianou (2005).

In a series of papers and two monographs, Boyarchenko and Levendorskii (2002a, 2002b, 2002c, 2005, 2006, 2007) developed a new approach to pricing of barrier and American options based on the operator form of the Wiener-Hopf method. The operator form is a standard analytical tool for solution of boundary problems for pseudo-differential equations; the new element is the interpretation of the factors as the expected present value operators (EPV-operators) operators which calculate the (discounted) expected present values of streams of payoffs under supremum and infimum processes. This interpretation allows one to guess the optimal exercise boundary quite naturally and give a simple proof of optimality. Boyarchenko and Levendorskii (2002a, b, c) calculated the prices of American options in Carr's randomization approximation Carr (1998) (equivalent to the analytic method of lines Carr and Faguet (1994)); a simplified version of the same procedure can be applied for pricing barrier options. However, no efficient numerical realization of the action of EPV-operators was suggested; later, Levendorskii (2004a) constructed a very accurate and fast realization of the pricing procedure for the American put options under diffusions with exponentially distributed jumps, and Kudryavtsev and Levendorskii (2006) derived an analytic approximation to prices of the first touch digitals under Normal Inverse Gaussian model.

In the present paper, we construct two efficient numerical methods for pricing barrier option under wide classes of Lévy processes; in the following publications, we will apply these methods to American options. Both methods start with time discretization, which can interpreted as Carr's randomization Carr (1998). A sequence of stationary boundary problems for a PDO on the line 
results. Problems of the sequence are solved by backward induction. The same sequence of problems arises in $\mathrm{CV}$-method and in the method constructed in Levendorskii et al (2006) (we will refer to the latter as FDS-method). Differences among the four methods appear at the next step, when the infinitesimal generator of the process or the inverse of the operator that solves the boundary problem must be approximated. Generally, an approximation of the inverse can be expected to perform better, and the fastest of the two methods constructed in the paper is based on an efficient approximation of the Wiener-Hopf factors in the exact formula for the solution. CV-method, FDS-method and the second method constructed in the paper use a decomposition of the operator into the leading part and the subordinate part but these decompositions are fundamentally different. A general comparison of CV-method, FDS-method and the two new methods is given Section 3 In Section 4 approximations of factors in the Wiener-Hopf factorization formula is discussed in more detail. In Section 5. we produce numerical examples, and compare the results obtained by the four methods. Section [6 concludes. In Section 2] we list the necessary facts of the theory of Lévy processes, explain the relation between Lévy processes and pseudo-differential operators (PDO) and recall the Wiener-Hopf factorization. We also explain crucial properties of prices of barrier options, that is, solutions of boundary problems for the generalized Black-Scholes equation, and properties of solutions of stationary Black-Scholes equations, which arise after time discretization. These properties are necessary to explain why CV-method may produce tangible errors unless a dozen hours of CPU time and more is spent.

\section{Lévy processes and barrier options}

\subsection{General definitions}

A Lévy process is a process with stationary independent increments (for general definitions, see e.g. Sato (1999)). A Lévy process may have a Gaussian component and/or pure jump component. The latter is characterized by the density of jumps, which is called the Lévy density. We denote it by $F(d y)$. A Lévy process can be completely specified by its characteristic exponent, $\psi$, definable from the equality $E\left[e^{i \xi X(t)}\right]=e^{-t \psi(\xi)}$ (we confine ourselves to the one-dimensional case). The characteristic exponent is given by the Lévy-Khintchine formula:

$$
\psi(\xi)=\frac{\sigma^{2}}{2} \xi^{2}-i \mu \xi+\int_{-\infty}^{+\infty}\left(1-e^{i \xi y}+i \xi y \mathbf{1}_{|y| \leq 1}\right) F(d y),
$$

where $\sigma^{2}$ is the variance of the Gaussian component, and $F(d y)$ satisfies

$$
\int_{\mathbf{R} \backslash\{0\}} \min \left\{1, y^{2}\right\} F(d y)<+\infty .
$$

If the jump component is a process of finite variation, equivalently, if

$$
\int_{\mathbf{R} \backslash\{0\}} \min \{1,|y|\} F(d y)<+\infty,
$$

then (11) can be simplified

$$
\psi(\xi)=\frac{\sigma^{2}}{2} \xi^{2}-i \mu \xi+\int_{-\infty}^{+\infty}\left(1-e^{i \xi y}\right) F(d y)
$$


with a different $\mu$, and the new $\mu$ is the drift of the gaussian component.

Assume that under a risk-neutral measure chosen by the market, the stock has the dynamics $S_{t}=e^{X_{t}}$. Then we must have $E\left[e^{X_{t}}\right]<+\infty$, and, therefore, $\psi$ must admit the analytic continuation into a strip $\operatorname{Im} \xi \in(-1,0)$ and continuous continuation into the closed strip $\operatorname{Im} \xi \in[-1,0]$. Further, if the riskless rate, $r$, is constant, and the stock does not pay dividends, then the discounted price process must be a martingale. Equivalently, the following condition must hold

$$
r+\psi(-i)=0
$$

which can be used to express $\mu$ via the other parameters of the Lévy process:

$$
\mu=r-\frac{\sigma^{2}}{2}+\int_{-\infty}^{+\infty}\left(1-e^{y}+y \mathbf{1}_{|y| \leq 1}\right) F(d y) .
$$

The infinitesimal generator of $X$, denote it $L$, is an integro-differential operator which acts as follows:

$$
L u(x)=\frac{\sigma^{2}}{2} u^{\prime \prime}(x)+\mu u^{\prime}(x)+\int_{-\infty}^{+\infty}\left(u(x+y)-u(x)-y \mathbf{1}_{|y| \leq 1} u^{\prime}(x)\right) F(d y) .
$$

\subsection{Lévy processes and PDO}

The infinitesimal generator $L$ can be represented as a pseudo-differential operator (PDO) with the symbol $-\psi(\xi): L=-\psi(D)$. Recall that a PDO $A=a(D)$ acts as follows:

$$
A u(x)=(2 \pi)^{-1} \int_{-\infty}^{+\infty} e^{i x \xi} a(\xi) \hat{u}(\xi) d \xi
$$

where $\hat{u}$ is the Fourier transform of a function $u$ :

$$
\hat{u}(\xi)=\int_{-\infty}^{+\infty} e^{-i x \xi} u(x) d x .
$$

Note that the inverse Fourier transform in (8) is defined in the classical sense only if the symbol $a(\xi)$ and function $\hat{u}(\xi)$ are sufficiently nice. In general, one defines the (inverse) Fourier transform, $F_{x \rightarrow \xi}$ (the subscript means that a function defined on the $x$-space becomes a function defined on the dual $\xi$ space), and the inverse Fourier transform, $F_{\xi \rightarrow x}^{-1}$ by duality; in many cases, in particular, in the context of pricing of put and call options, one can use the classical definition of the integral but integrate in (8) not over the real line but along an appropriate line or contour in the complex plane. See, e.g., Boyarchenko and Levendorkii (2002a-c) for details and examples.

Formally, the action of a PDO $A$ with the constant symbol $a(\xi)$ can be described as the composition

$$
A u(x)=F_{\xi \rightarrow x}^{-1} a(\xi) F_{x \rightarrow \xi} u(x)
$$

of the Fourier transform, multiplication by a given function (symbol) and the inverse Fourier transform. All three operations can be performed very fast, and the built-in procedures for these operations are readily available. If the functions $u$ and $a$ are represented as arrays suitable for application of the Fast 
Fourier Transform and inverse Fast Fourier Transfrom algorithms (FFT and iFFT), then (9) can be programmed as

$$
A u=i F F T(a . *(F F T(u))) .
$$

We would like to stress the fact that this is the representation of the infinitesimal generator in the form of a PDO that allows one to establish quite easily main properties of $L$ and solutions of boundary value problems. The importance of the interpretation of integro-differential operators as PDOs was recognized in 1960's, and was one of the main motivation for the theory of PDO itself - see e.g. Eskin (1973) and Hörmander (1985). Furthermore, the PDO- interpretation of the Wiener-Hopf factorization allows us to write the solution of the boundary problems on the line that arise after time discretization of the boundary problem for the barrier option in the form similar to (9), with several multiplication operators and copies of the Fourier transform and inverse Fourier transform. This form of the solution, together with an efficient approximate procedure of calculation of the Wiener-Hopf factors, leads to a very fast and accurate pricing algorithm for barrier options.

\subsection{Regular Lévy processes of exponential type}

In order that the PDO technique were not complicated and solutions to boundary problems be regular, the symbols should be sufficiently nice. For PDO with constant symbols (meaning: symbols independent of $x$ ), a convenient condition is: the symbol admits a representation

$$
a(\xi)=a_{m}(\xi)+O\left(|\xi|^{m_{1}}\right), \quad \text { as } \quad \xi \rightarrow \infty
$$

where $a_{m}$ is positively homogeneous of degree $m$, and $m_{1}<m$ (for PDO with "variable" symbols $a(x, \xi)$, additional conditions on the derivatives of the symbol are needed). The $m$ is called the order of PDO $a(D)$, and $a_{m}$ is called the principal symbol of $a(D)$. If it is necessary to consider the action of a PDO in spaces with exponential weights, then the representation (10) must be valid in an appropriate strip of the complex plane (in the multi-dimensional case, in a tube domain in $\mathbf{C}^{n}$ ) - see e.g. Eskin (1973), Barndorff-Nielsen and Levendorskii (2001) and Boyarchenko and Levendorskii (2002b). Essentially, these two properties (the characteristic exponent is analytic in a strip, and (10) is valid in the strip) are used in Boyarchenko and Levendorskii (1999, 2000, 2002a-c) to introduce the class of RLPE in terms of the characteristic exponent; the other definition starts with the Lévy density.

Loosely speaking, a Lévy process $X$ is called a Regular Lévy Process of Exponential type (RLPE) if its Lévy density has a polynomial singularity at the origin and decays exponentially at the infinity (see Boyarchenko and Levendorskii (1999, 2000, 2002a-c). An almost equivalent definition is: the characteristic exponent is analytic in a strip $\operatorname{Im} \xi \in\left(\lambda_{-}, \lambda_{+}\right)$, continuous up to the boundary of the strip, and admits the representation

$$
\psi(\xi)=-i \mu \xi+\phi(\xi)
$$

where $\phi(\xi)$ stabilizes to a positively homogeneous function at the infinity:

$$
\phi(\xi) \sim c_{ \pm}|\xi|^{\nu}, \quad \text { as } \operatorname{Re} \xi \rightarrow \pm \infty, \quad \text { in the strip } \operatorname{Im} \xi \in\left(\lambda_{-}, \lambda_{+}\right)
$$


where $c_{ \pm}>0$. "Almost" means that the majority of classes of Lévy processes used in empirical studies of financial markets satisfy conditions of both definitions. These classes are: Brownian motion, Kou's model (Kou (2002)), Hyperbolic processes (Eberlein and Keller (1995), Eberlein at all (1998)), Normal Inverse Gaussian processes and their generalization (Barndorff-Nielsen (1998) and Barndorff-Nielsen and Levendorskï (2001)), and extended Koponen's family. Koponen (1995) introduced a symmetric version; Boyarchenko and Levendorskii $(1999,2000)$, gave a non-symmetric generalization; later a subclass of this model appeared under the name CGMY-model in Carr et al. (2002), and Boyarchenko and Levendorskii (2002a-c) used the name KoBoL family. The two important exceptions are Variance Gamma Processes (VGP; see, e.g., Madan et al. (1998)) and stable Lévy processes. VGP satisfy the conditions of the first definition but not the second one, since the characteristic exponent behaves like const $\cdot \ln |\xi|$, as $\xi \rightarrow \infty$, and the characteristic exponent of a stable Lévy process does not admit the analytic continuation into a strip adjacent to the real line.

Example 2.1. The characteristic exponent of a pure jump KoBoL process of order $\nu \in(0,2), \nu \neq 1$ is given by

$$
\psi(\xi)=-i \mu \xi+c \Gamma(-\nu)\left[\lambda_{+}^{\nu}-\left(\lambda_{+}+i \xi\right)^{\nu}+\left(-\lambda_{-}\right)^{\nu}-\left(-\lambda_{-}-i \xi\right)^{\nu}\right],
$$

where $c>0, \mu \in \mathbf{R}$, and $\lambda_{-}<-1<0<\lambda_{+}$. Formula (13) is derived in Boyarchenko and Levendorskii $(1999,2000,2002 b)$ from the Lévy-Khintchine formula with the Lévy densities of negative and positive jumps, $F_{\mp}(d y)$, given by

$$
F_{\mp}(d y)=c e^{\lambda_{ \pm} y}|y|^{-\nu-1} d y .
$$

Note that Boyarchenko and Levendorskii $(2000,2002 b)$ consider a more general version with $c_{ \pm}$instead of $c$, as well as the case $\nu=1$ and cases of different exponents $\nu_{ \pm}$). If $\nu \geq 1$ or $\mu=0$, then the order of the KoBoL process equals to the order of the infinitesimal generator as PDO, but if $\nu<1$ and $\mu \neq 0$, then the order of the process is $\nu$, and the order of the PDO $-L=\psi(D)$ is 1 . Hyperbolic and normal inverse Gaussian processes are RLPEs of order 1, and the generalization of normal inverse processes constructed in Barndorff-Nielsen and Lévendorskii (2001) contains processes of any order $\nu \in(0,2)$. If there is a diffusion component, the order of the process is 2 . The difference between the definition in terms of the Lévy density and the one in terms of the characteristic exponent is apparent in the case of a VGP without the diffusion component: the Lévy density is given by (14) with $\nu=0$ but the characteristic exponent involves a logarithmic function. Hence, the infinitesimal generator of a VGP is not of order 0 .

Example 2.2. A normal inverse Gaussian process (NIG) can be described by the characteristic exponent of the form

$$
\psi(\xi)=-i \mu \xi+\delta\left[\left(\alpha^{2}-(\beta+i \xi)^{2}\right)^{1 / 2}-\left(\alpha^{2}-\beta^{2}\right)^{1 / 2}\right],
$$

where $\alpha>|\beta|>0, \delta>0$ and $\mu \in \mathbf{R}$.

\subsection{The Wiener-Hopf factorization}

There are several forms of the Wiener-Hopf factorization. The Wiener-Hopf factorization formula used in probability reads:

$$
E\left[e^{i \xi X_{T}}\right]=E\left[e^{i \xi \bar{X}_{T}}\right] E\left[e^{i \xi \underline{X}_{T}}\right], \quad \forall \xi \in \mathbb{R},
$$


where $T \sim \operatorname{Exp} q$, and $\bar{X}_{t}=\sup _{0 \leq s \leq t} X_{s}$ and $\underline{X}_{t}=\inf _{0 \leq s \leq t} X_{s}$ are the supremum and infimum processes. Introducing the notation

$$
\begin{aligned}
& \phi_{q}^{+}(\xi)=q E\left[\int_{0}^{\infty} e^{-q t} e^{i \xi \bar{X}_{t}} d t\right]=E\left[e^{i \xi \bar{X}_{T}}\right], \\
& \phi_{q}^{-}(\xi)=q E\left[\int_{0}^{\infty} e^{-q t} e^{i \xi \underline{X}_{t}} d t\right]=E\left[e^{i \xi \underline{X}_{T}}\right]
\end{aligned}
$$

we can write (16) as

$$
\frac{q}{q+\psi(\xi)}=\phi_{q}^{+}(\xi) \phi_{q}^{-}(\xi) .
$$

Equation (19) is a special case of the Wiener-Hopf factorization of the symbol of a PDO. In applications to Lévy processes, the symbol is $q /(q+\psi(\xi))$, and the PDO is $\mathcal{E}:=q /(q-L)=q(q+\psi(D))^{-1}$ : the normalized resolvent of the process $X_{t}$ or, using the terminology of Boyarchenko and Levendorskii (2005, $2006,2007)$, the expected present value operator (EPV-operator) of the process $X_{t}$. The name is due to the observation that, for a stream $g\left(X_{t}\right)$,

$$
\mathcal{E} g(x)=E\left[\int_{0}^{+\infty} q e^{-q t} g\left(X_{t}\right) d t \mid X_{0}=x\right] .
$$

The factors $\phi_{q}^{ \pm}(\xi)$ also admit interpretation as the symbols of the EPV-operators $\mathcal{E}^{ \pm}=\phi_{q}^{ \pm}(D)$ under supremum and infimum processes

$$
\begin{aligned}
& \mathcal{E}^{+} g(x):=q E\left[\int_{0}^{\infty} e^{-q t} g\left(\bar{X}_{t}\right) d t \mid X_{0}=x\right] \\
& \mathcal{E}^{-} g(x):=q E\left[\int_{0}^{\infty} e^{-q t} g\left(\underline{X}_{t}\right) d t \mid X_{0}=x\right] .
\end{aligned}
$$

One of the basic observations in the theory of PDO is that the product of symbols corresponds to the product of operators. In our case, it follows from (19) that

$$
\mathcal{E}=\mathcal{E}^{+} \mathcal{E}^{-}=\mathcal{E}^{-} \mathcal{E}^{+}
$$

as operators in appropriate function spaces. To study the regularity of the price of a barrier option or first touch digital, one needs a somewhat more general form of the Wiener-Hopf factorization, with $q=i \eta$, where $\eta$ runs over an appropriate contour in the complex plane. See Boyarchenko and Levendorskii $(2002 \mathrm{~b}, \mathrm{c})$ and Kudryavtsev and Levendorskii (2006).

\subsection{The generalized Black-Scholes equation for barrier op- tions}

For the sake of brevity, consider the down-and-out put option without rebate, with strike $K$, maturity $T$ and barrier $H<K$, on a non-dividend paying stock. Let $V\left(t, X_{t}\right)$ be the option value at time $t<T$ and spot price $e^{X_{t}}$. Boyarchenko and Levendorskii $(2002 \mathrm{~b}, \mathrm{c})$ proved that in the region $x>h: \log H, t<T$, where the option remains alive, the function $V$ satisfies the generalized Black-Scholes equation

$$
\left(\partial_{t}+L-r\right) V(t, x)=0, \quad t<T, x>h,
$$


subject to the terminal condition

$$
V(T, x)=\left(K-e^{x}\right)_{+}
$$

and boundary condition

$$
V(t, x)=0, \quad t<T, x \leq h .
$$

In addition, $V$ must be bounded. Equation (21) was derived under a weak regularity condition: the process $\left(t, X_{t}\right)$ in $2 \mathrm{D}$ satisfies (ACP)-condition (for the definition, see, e.g., Sato (1999)). Note that (ACP) condition is satisfied if the process $X_{t}$ has the transition density. Equation (21) is understood in the sense of the theory of generalized functions: for any infinitely smooth function $u$ with compact support $\operatorname{supp} u \subset(-\infty, T) \times(h,+\infty)$,

$$
\left(V,\left(-\partial_{t}+\tilde{L}-r\right) u\right)_{L_{2}}=0,
$$

where $\tilde{L}=\bar{\psi}(D)$ is the infinitesimal generator of the dual process $(\bar{\psi}(\xi)$ is the complex-conjugate to $\psi(\xi)$ ). One cannot state that the boundary problem (21)(23) has a unique bounded solution for an arbitrary Lévy process. However, if the symbol $i \eta-\psi(\xi)-r$ of the operator $\partial_{t}+L-r$ is sufficiently regular, then the general technique of the theory of PDO can be applied to show that, first, a continuous bounded solution is unique, and, second, the solution is of class $C^{\infty}$ in $(-\infty, T] \times((h, \log K) \cup(\log K,+\infty))$. For details, see Boyarchenko and Levendorskiï (2002b,c) and Kudryavtsev and Levendorskii (2006). Boyarchenko and Levendorskii (2002b) showed that, typically, the derivative of the solution w.r.t. $x$ is not smooth as $x \downarrow h$ (in a neighborhood of $(t, x)=(T, \log K)$, the regularity of the price of the barrier option is the same as the regularity of the corresponding European option). To be more specific, they proved for the first-touch digitals that the derivative of the option price w.r.t. $x$ behaves as $|x-h|^{\nu_{-}-1}$ as $x \downarrow h$, where $-\nu_{-} \in(-1,0)$ is the order of the decay of the symbol $\phi_{q}^{-}(\xi)$ as $\xi \rightarrow \infty$. Using the same technique, one can show that the prices of barrier options enjoy the same property. If the process has the gaussian component, then $\nu_{-}=1$, and, therefore, the first derivative of the option price is bounded as $x \downarrow h$ (but, in the presence of the jump component of infinite variation, one cannot guarantee that the second derivative is bounded). For an RLPE of order $\nu \in(1,2), \nu_{-}=\nu / 2$ if $c_{+}=c_{-}$; if $c_{+} \neq c_{-}$and for processes of order 1 (e.g., Normal Inverse Gaussian processes and Hyperbolic processes), any $\nu_{-} \in(0,1)$ is possible but not $\nu_{-}=1$. In addition, if the "drift coefficient" $\mu$ is close to 0 , then $\nu_{-}$is close to $\nu / 2$. For processes of order less than 1 , the situation becomes even more involved - see Boyarchenko and Levendorskii (2002b) and Levendorskii (2004b) for details. Thus, in general, if there is no gaussian component, then the first derivative of the option price is unbounded as the spot price of the underlying approaches the barrier. Boyarchenko and Levendorskii (2002b) do not study the behavior of the second derivative but it can be shown that this derivative is unbounded even if the gaussian component is present but the jump component is of infinite intensity. The implication is that one cannot use the Taylor expansion of order 2 to approximate the option value near the barrier; hence, one cannot hope that the approximation of small jumps by an additional diffusion component will be reasonably accurate near the barrier. We will see that the error of this approximation can be felt fairly far from the barrier. 


\section{Four methods}

\subsection{Analytic method of lines or Carr's randomization}

As an example, consider the down-and-out put option with barrier $H=e^{h}<K$, zero rebate and payoff $G\left(X_{T}\right)=\left(K-e^{X_{T}}\right)+$ at expiry. We divide $[0, T]$ into $N$ time steps of length $\Delta t=T / N$, set $V^{N}(x)=G(x), q=r+1 / \Delta t$, discretize the time derivative, and find $V^{s}$, an approximation to the price of the barrier option at time $t_{s}=s \Delta t, s=N-1, N-2, \ldots$, as a unique bounded solution of the boundary problem

$$
\begin{aligned}
q^{-1}(q-L) V^{s}(x) & =\frac{1}{q \Delta t} V^{s+1}(x), \quad x>h, \\
V^{s}(x) & =0, \quad x \leq h .
\end{aligned}
$$

A general class of boundary problems that contains the problem (25) - 26) was studied in Boyarchenko and Levendorskii (2002a, b) and Levendorskii (2004b). It was shown that the unique bounded solution is given by

$$
V^{s}=\frac{1}{q \Delta t} \mathcal{E}^{-} \mathbf{1}_{[h,+\infty)} \mathcal{E}^{+} V^{s+1},
$$

where $\mathbf{1}_{[h,+\infty)}$ is the indicator function of $[h,+\infty)$. If the analytic expressions for the Wiener-Hopf factors $\phi_{q}^{ \pm}(\xi)$ are available, one can calculate $V^{s}$ quite easily using FFT, iFFT and multiplication operators by functions $\phi_{q}^{ \pm}$and $\mathbf{1}_{[h,+\infty)}$ :

$$
V^{s}(x)=\frac{1}{q \Delta t} F_{\xi \rightarrow x}^{-1} \phi_{q}^{-}(\xi) F_{x \rightarrow \xi} \mathbf{1}_{[h,+\infty)}(x) F_{\xi \rightarrow x}^{-1} \phi_{q}^{+}(\xi) F_{x \rightarrow \xi} V^{s+1}(x) .
$$

The explicit command sequence is

$$
V^{s}=1 /(q * \Delta t) * i F F T\left(\phi_{q}^{-} \cdot * F F T\left(\mathbf{1}_{[h,+\infty)} \cdot * i F F T\left(\phi_{q}^{+} \cdot * F F T\left(V^{s+1}\right)\right)\right)\right) .
$$

Here $*$ is the multiplication-by-scalar, and $*$ is the element-wise multiplication of arrays that represent the functions in question after discretization and truncation of the state space and the dual space.

We explain why $V^{s}(x)$ is, typically, irregular at the boundary using an example of Normal Inverse Gaussian processes, without the "drift" term. The characteristic exponent is of the form (15) with $\mu=0$, and it can be shown that the factors $\phi_{q}^{ \pm}(\xi)$ in the Wiener-Hopf factorization formula (19) have the following asymptotics as $\xi \rightarrow \pm \infty$ :

$$
\phi_{q}^{ \pm}(\xi) \sim e^{ \pm i \pi / 4}\left(q^{-1} \delta|\xi|\right)^{-1 / 2}
$$

Generically, function $w:=\mathcal{E}^{+} V^{s+1}$ has no zero at $x=h$, therefore, the Fourier transform of the function $w_{1}:=\mathbf{1}_{[h,+\infty)} w$ is of the form $e^{-i h \xi} u(\xi)$, where $u(\xi)$ decays at infinity not faster than $|\xi|^{-1}$. Since $\phi_{q}^{-}(\xi)$ is the symbol of the EPV operator $\mathcal{E}^{-}=\phi_{q}^{-}(D)$, and the asymptotics (30) holds, we apply the Fourier transform to (27) and conclude that

$$
\hat{V}^{s}(\xi)=\frac{1}{q \Delta t} e^{-i h \xi} \phi_{q}^{-}(\xi) u(\xi)
$$


decays at infinity not faster than $|\xi|^{-3 / 2}$. Therefore, the Fourier transform of the derivative $\left(V^{s}\right)^{\prime}$ decays at infinity not faster than $|\xi|^{-1 / 2}$, and does not oscillate at $x=h$. This implies that the derivative is unbounded as $x \downarrow h$. A more detailed study shows that

$$
\left(V^{s}\right)^{\prime}(x) \sim \text { const } \cdot(x-h)^{-1 / 2} \quad \text { as } x \downarrow h .
$$

See Boyarchenko and Levendorskii (2002a, b).

\subsection{CV-method}

The infinitesimal generator of a Lévy process can be represented as a sum of the Gaussian and pure-jump components, denote them by $L_{G}$ and $L_{J}$, respectively. In $\mathrm{CV}$-method, the part of the infinitesimal generator corresponding to jumps of size $|y|<\epsilon$, where $\epsilon>0$ is a (small) parameter of the method, is replaced with a differential operator of the second order, call it $L_{G, \epsilon} ;$ jumps of a large size $|y| \geq$ $M$ are truncated. Denoting by $L_{J, \epsilon, M}$ the part of the infinitesimal generator corresponding to jumps of size $\epsilon \leq|y| \leq M$, one obtains the approximation

$$
L \approx L_{G}+L_{G, \epsilon}+L_{J, \epsilon, M}
$$

Equation (25) is replaced by

$$
\left(q-L_{G}-L_{G, \epsilon}\right) V^{s}(x)=\left(\frac{1}{\Delta t}+L_{J, \epsilon, M}\right) V^{s+1}(x), \quad x>h,
$$

that is, an explicit-implicit scheme is used. Since $L_{G}+L_{G, \epsilon}$ is the infinitesimal generator of a Brownian motion, the solution of the boundary problem (32), (26) is of class $C^{2}$ up to the boundary. Hence, if the initial process has no gaussian component, then, in a neighborhood of the boundary, the option value $V^{s}$ calculated using $\mathrm{CV}$-method differs significantly from the correct option value. The numerical examples in Section [5] show that, for realistic parameter values, this neighborhood can be quite sizable.

A somewhat different but, essentially, very close explanation of errors of the approximation (31) follows from the standard perturbation considerations. If $L_{G}=0$, then $L$ is the PDO of order $\nu<2$. The approximation (31)-(32) imply that the second order operator $L_{G, \epsilon}$ is used as the principal part of $L$. But the order of the former, 2, is higher than the order of the latter, $\nu<2$. The basic analytic intuition says that one cannot use an operator of the order higher than the order of the initial operator, as the leading operator: the orders must be equal. Moreover, it is preferable that the difference between these two operators, call them $A$ and $A_{0}$, be of smaller order. This means that the symbol of $A-A_{0}$ must grow slower than the symbol of $A$ as $\xi \rightarrow \infty$. In the case of an operator with large parameter, here, $q$, the difference must grow slower as $q \rightarrow+\infty$ as well 11 A decomposition of $A:=q^{-1}(q-L)$ with this property is used as the basis of the first new method constructed in the paper.

\footnotetext{
${ }^{1}$ It is even better to have a relative decay as $|\xi|+q \rightarrow \infty$ but, for operators under consideration, one cannot find a leading part which admits a simple factorization and enjoys this strong property.
} 


\subsection{FDS-method}

In Levendorskii et al. (2006), a more accurate procedure applicable for processes of order $\nu<1$ is developed and applied to the pricing problem of American options with finite time horizon. Certainly, essentially the same procedure can be applied for pricing of barrier options: in the case of barrier options, the boundary is fixed in advance and so it is unnecessary to choose it at each time step. FDS-method differs from CV-method in two respects, which lead to a better accuracy. First, the part of the infinitesimal generator corresponding to jumps of size $|y|<\epsilon$, where $\epsilon>0$ is a (small) parameter of the method, is replaced with a differential operator of the first order (additional drift component rather than diffusion component), call it $L_{D, \epsilon}$; jumps of a large size $|y| \geq M$ are truncated. Thus, we have the following analog of (31):

$$
L \approx \mu \partial_{x}+L_{D, \epsilon}+L_{J, \epsilon, M}
$$

The advantage of the approximation (33) as compared to (31) (with $L_{G}=$ 0 ) is that the order of the operator remains the same, which makes errors of the approximation smaller; an approximation by an additional drift becomes possible because for processes of order $\nu<1$, the density $y F(d y)$ is integrable. The second improvement in accuracy is more general and can be applied to modify CV-model as well, at the cost of additional computational time. Instead of the straightforward explicit-implicit scheme as in CV-method, FDS can be characterized as an explicit scheme with an iteration procedure at each time step, which utilizes the decomposition of the operator. For each time step, instead of (32), we solve a sequence of problems

$$
\left(q-\mu \partial_{x}-L_{D, \epsilon}\right) V_{n+1}^{s}(x)=\frac{1}{\Delta t} V^{s+1}(x)+L_{J, \epsilon, M} V_{n}^{s}(x), \quad x>h,
$$

$n=0,1,2, \ldots$, where $V_{0}^{s}=V^{s+1}$; calculations in the cycle in $n$ are stopped when the $l_{\infty}$-norm of $V_{n+1}^{s}-V_{n}^{s}$ becomes smaller than the chosen error tolerance.

Numeric solution to the problems (32) and (34) requires discretization in $x$-space: the derivatives are replaced by finite differences, and integral term is approximated by discrete sum.

Remark 3.1. The types of errors of CV-method and FDS-method are similar but, for processes of order $\nu<1$, the order of errors of the former can be expected to be much larger than the order of errors of the latter - provided the same $M, \epsilon$ and the grids in $t$ and $x$-space are chosen. Thus, we can use FDS (which is slower) as a benchmark to test the accuracy of CV for processes of order $\nu<1$.

\subsection{Iterative Wiener-Hopf method (IWH-method)}

If explicit and convenient for numerical realization formulas for the factors $\phi_{q}^{ \pm}(\xi)$ are available, then the option value given by (27) can be calculated easily using the fast Fourier transform. If the factors decay at infinity fast, then FFT and iFFT work accurately and fast. Unfortunately, apart from a handful of simple cases: Brownian motion, diffusions with exponentially distributed jumps and spectrally negative (or positive) processes, simple formulas for $\phi_{q}^{ \pm}(\xi)$ are not available. To avoid this difficulty, we decompose $A:=q^{-1}(q-L)$ into the sum 
of a simple operator, call it $A_{q, 0}$, that admits an analytical factorization, and the subordinate operator, call it $A_{q, \text { sub }}$. This is the standard trick in the theory of PDE and PDO with parameter. If the subordinate operator, $A_{q, \text { sub }}$, is, indeed, subordinate to the leading part $A_{q, \nu}$, then the decomposition works well. After that, an appropriate iterative scheme is designed. As an example, consider a NIG process without drift. The characteristic exponent is of the form

$$
\psi(\xi)=\delta\left[\left(\alpha^{2}-(\beta+i \xi)^{2}\right)^{1 / 2}-\left(\alpha^{2}-\beta^{2}\right)^{1 / 2}\right],
$$

therefore, as the leading part of the symbol $a(q, \xi):=q^{-1}(q+\psi(\xi))$, it seems natural to take $1+(\delta / q)|\xi|$. However, this function does not admit a simple factorization. So, instead, we take the function $a_{q, 0}(\xi):=\left(1+i q^{-1} \delta \xi\right)^{1 / 2}(1-$ $\left.i q^{-1} \delta \xi\right)^{1 / 2}$, which is already factorized and enjoys an important property

$$
\left(a(q, \xi)-a_{0}(q, \xi)\right) / a_{0}(q, \xi) \rightarrow 0
$$

as $|\xi| \rightarrow \infty$ (for $q$ fixed) and as $q \rightarrow+\infty$ (for $\xi \in \mathbb{R}$ fixed). Thus, as the leading operator, we take $\left.A_{q, 0}=\left(1-(\delta / q) \partial_{x}^{2}\right)\right)^{1 / 2}$, which admits the factorization

$$
A_{q, 0}=\left(1+i(\delta / q) D_{x}\right)^{1 / 2}\left(1-i(\delta / q) D_{x}\right)^{1 / 2} .
$$

Denote by $\phi_{q, 0}^{ \pm}$the symbols of the factors in the decomposition of $A_{q, 0}$ :

$$
\phi_{q, 0}^{ \pm}(\xi)=\left(1 \mp i q^{-1} \delta \xi\right)^{1 / 2}
$$

(note that one must have sign $\mp$ on the RHS because the "plus"-factor in the Wiener-Hopf factorization formula admits the analytic continuation into the upper half-plane $\operatorname{Im} \xi>0)$, set $A_{q, s u b}=q^{-1}\left(q-L_{0}\right)-A_{q, 0}$ and

$$
a_{q, s u b}(\xi)=q^{-1}(q-\psi(\xi))-\phi_{q, 0}^{+}(\xi) \phi_{q, 0}^{-}(\xi),
$$

and rewrite (40) in the form convenient for a natural iteration method:

$$
A_{q, 0} V^{s}(x)=\frac{1}{q \Delta t} V^{s+1}(x)+A_{q, s u b} V^{s}(x), \quad x>h .
$$

The iteration procedure is as follows (we formulate it for a general terminal payoff function $G\left(X_{T}\right)$; for the standard payoffs, the calculation at step III below can be made analytically, without resorting to FFT):

I. Choose finite grids $x_{j}=x_{1}+\lambda j$ in $x$-space and $\xi_{k}=\xi_{1}+k \zeta$ in $\xi$-space, $j, k=1, \ldots, 2^{m}$, appropriate for the FFT and iFFT algorithms.

II. Calculate $\phi_{q, 0}^{ \pm}\left(\xi_{k}\right)$ and $a_{q, s u b}\left(\xi_{k}\right), k=1, \ldots, 2^{m}$, and $V^{N}\left(x_{j}\right)=\left(K-e_{j}^{x}\right)_{+}$ and $\mathbf{1}_{[h,+\infty)}\left(x_{j}\right), j=1, \ldots, 2^{m}$.

III. Calculate the Fourier transform $\hat{V}^{N}$ using FFT.

IV. In the cycle in $s=N-1, N-2, \ldots, 0$, assign $V^{s, 0}=V^{s+1}$ and $\hat{V}^{s, 0}=\hat{V}^{s+1}$, and then, in the cycle in $n=1,2, \ldots$,

(1) calculate $\hat{u}_{n}:=(q \Delta t)^{-1} * \hat{V}^{s+1}+a_{q, s u b} * \hat{V}^{s, n-1}$; 
(2) apply the composition of FFT, iFFT and multiplication-by-function operators to calculate

$$
\hat{V}^{s, n}(\xi)=\phi_{q, 0}^{-}(\xi) F_{x \rightarrow \xi} \mathbf{1}_{[h,+\infty)}(x) F_{\xi \rightarrow x}^{-1} \phi_{q, 0}^{+}(\xi) \hat{u}_{n}(\xi)
$$

at the points of the grid in $\xi$-space;

(3) calculate the upper bound $\epsilon_{s, n}:=\left\|\hat{V}^{s, n}-\hat{V}^{s, n-1}\right\|_{l_{1}}$ for $\| V^{s, n}-$ $V^{s, n-1} \|_{l_{\infty}}$.

If $\epsilon_{s, n}$ is smaller than the chosen error tolerance, stop.

V. calculate $V^{0}(x)=\left(F_{\xi \rightarrow x}^{-1} \hat{V}^{0}\right)(x)$ at the points of the grid in $x$-space.

\subsection{Implicit-explicit Wiener-Hopf method}

Similarly to CV-method, we can simplify the scheme above and, instead of the iteration procedure at each time step, use the implicit-explicit procedure

$$
A_{q, 0} V^{s}(x)=\frac{1}{q \Delta t} V^{s+1}(x)+A_{q, s u b} V^{s+1}(x), \quad x>h .
$$

In the algorithm above, steps I-III remain the same but step IV becomes

IV. In the cycle in $s=N-1, N-2, \ldots, 0$,

(1) calculate $\hat{u}^{s}:=(q \Delta t)^{-1} * \hat{V}^{s+1}+a_{q, s u b} * \hat{V}^{s+1}$;

(2) apply the composition of FFT, iFFT and multiplication-by-function operators to calculate

$$
\hat{V}^{s}(\xi)=\phi_{q, 0}^{-}(\xi) F_{x \rightarrow \xi} \mathbf{1}_{[h,+\infty)}(x) F_{\xi \rightarrow x}^{-1} \phi_{q, 0}^{+}(\xi) \hat{u}^{s}(\xi)
$$

at the points of the grid in $\xi$-space, and then $V^{s}(x)=\left(F_{\xi \rightarrow x}^{-1} \hat{V}^{s}\right)(x)$ at the points of the grid in $x$-space.

\subsection{IWH-method for more general processes}

If the "drift" $\mu \neq 0$, then either the construction of the leading operator becomes complicated: the orders of the factors become different (see Boyarchenko and Levendorskii $(2002 \mathrm{a}, \mathrm{b}))$ or the drift must be removed. If the "drift" is not large (in modulus) then the second course of action should be preferred. We have to return to the initial boundary problem (21) - 23) in continuous time, and change the variables $(t, x)=\left(t, x^{\prime}+\mu(t-T)\right)$. Then the terminal condition (22) does not change, boundary condition (23) becomes

$$
V\left(t, x^{\prime}\right)=0, \quad t<T, x^{\prime} \leq h-\mu(t-T),
$$

and the "drift term" in (21) disappears:

$$
\left(\partial_{t}+L^{0}-r\right) V\left(t, x^{\prime}\right)=0, \quad t<T, x^{\prime}>h-\mu(t-T),
$$

where $L^{0}=\phi(D)$ is the PDO with the symbol $\phi$. After the time discretization, we obtain a sequence of boundary problems

$$
\begin{aligned}
q^{-1}\left(q-L^{0}\right) V^{s}\left(x^{\prime}\right) & =\frac{1}{q \Delta t} V^{s+1}\left(x^{\prime}\right), \quad x^{\prime}>h_{s} \\
V^{s}\left(x^{\prime}\right) & =0, \quad x^{\prime} \leq h_{s}
\end{aligned}
$$


where $h_{s}=h-\mu\left(t_{s}-T\right)$. The modifications of the algorithm above are evident: one has to use different indicator functions $\mathbf{1}_{\left[h_{s},+\infty\right)}$ at different time steps.

Now consider a Lévy process whose Lévy exponent admits representation (11) - (12). The construction of the leading term depends on the order of the process, $\nu$, "drift", $\mu$, and constants $c_{ \pm}$in (12). For the sake of brevity, we consider the case $c_{+}=c_{-}=c$. Then, after the drift removal, we use the leading term

$$
L_{q, 0}^{0}=\left[1+i(c / q)^{1 / \nu} D_{x}\right]^{\nu / 2}\left[1-i(c / q)^{1 / \nu} D_{x}\right]^{\nu / 2} .
$$

For the case $c_{+} \neq c_{-}$, the reader can find similar factorizations in the case $c_{+} \neq c_{-}$in Boyarchenko and Levendorskii $(2002 \mathrm{~b})$. The algorithm is the same.

\subsection{Fast Wiener-Hopf factorization method (FWH-method)}

The last (and fastest) method uses the exact formula (28). Once sufficiently accurate approximations to the factors $\phi_{q}^{ \pm}$are constructed, the calculation of the option price becomes accurate and fast: faster than IWH-method because no iteration is involved and more accurate than $\mathrm{CV}$-method because the approximation does not imply that the option value is of class $C^{2}$ up to the boundary. The FWH-method enjoys an additional appealing feature: for arbitrary number of time steps, approximate formulas for $\phi_{q}^{ \pm}$are needed at the first and last steps in the cycle in $s$ only. At all intermediate steps, the exact analytic expression $q /(q+\psi(\xi)$ is used, and, therefore, almost all errors are errors of FFT and iFFT only. Indeed, for $s=N-1, N-2, \ldots, 0$, define

$$
W^{s}=\mathbf{1}_{[h ;+\infty)} \mathcal{E}^{+} V^{s+1} .
$$

Then

$$
V^{s}=(q \Delta t)^{-1} \mathcal{E}^{-} W^{s}(x) .
$$

Using the Wiener-Hopf factorization formula (20), we obtain that

$$
W^{s}=(q \Delta t)^{-1} \mathbf{1}_{[h ;+\infty)} \mathcal{E} W^{s+1},
$$

or, equivalently,

$$
\hat{W}^{s}(\xi)=(q \Delta t)^{-1} F_{x \rightarrow \xi} \mathbf{1}_{[h ;+\infty)} F_{\xi \rightarrow x}^{-1} \frac{q}{q+\psi(\xi)} \hat{W}^{s+1}(\xi) .
$$

Thus, if we are interested in the option value $V^{0}$ at the last step only, the algorithm becomes very simple:

1. On the chosen grid in $\xi$-space, calculate $\hat{V}^{N}(\xi)$, an approximation $\phi_{q, a}^{+}(\xi)$, and the array $w^{N}:=\phi_{q, a}^{+} * \hat{V}^{N}$.

2. Using the composition of iFFT, multiplication-by- $\mathbf{1}_{[h,+\infty)}$ and FFT, calculate

$$
\hat{W}^{N}=(q \Delta t)^{-1} F \mathbf{1}_{[h ;+\infty)} F^{-1} w^{N} .
$$

3. In the cycle in $s=N-1, N-2, \ldots, 0$, use the composition of iFFT, multiplication-by-1 $\mathbf{1}_{[h,+\infty)}$, multiplication-by- $q /(q+\psi(\xi))$ and FFT, calculate $\hat{W}^{s}$ in (45). 
4. On the chosen grid in $\xi$-space, calculate an approximation $\phi_{q, a}^{-}(\xi)$, and the array $\hat{V}^{0}=(q \Delta t)^{-1} * \phi_{q, a}^{-} * \hat{W}^{0}$.

5. Apply iFFT to calculate $V^{0}=F^{-1} \hat{V}^{0}$.

Remark 3.2. a) Depending on the type of the option under consideration, we choose real $\omega$, and apply the Fourier transform $F_{x \rightarrow \xi}$ and the inverse Fourier transform $F_{\xi \rightarrow x}^{-1}$ with $x$ living in $\mathbb{R}$ and $\xi$ living in $\mathbb{R}+i \omega$. Thus, a grid for $\xi$ is the grid $\xi_{j}=\eta_{j}+i \omega$ on the $\operatorname{line} \operatorname{Im} \xi=\omega$. For a put option, we may take any $\omega_{+}>0$ such that $\operatorname{Re}(q+\psi(\xi))>0$ in the strip $\operatorname{Im} \xi \in\left[0, \omega_{+}\right]$, and for a call option, $\omega_{-}<-1$ such that $\operatorname{Re}(q+\psi(\xi))>0$ in the $\operatorname{strip} \operatorname{Im} \xi \in\left[\omega_{-}, 0\right]$. For a down-and-out put option, we may and will take $\omega_{+}=0$. This will allow us to use the real Fast Fourier transform algorithm, which is faster and more accurate than FFT.

b) Approximation of the Fourier transform (resp., inverse Fourier transform) using FFT (resp., iFFT) involves two types of errors: truncation error and discretization error. For an RLPE, the truncation error for FFT can be made small very easily because the put option price decays exponentially as $x \rightarrow+\infty$ (we may consider the put options only because of the call-put pairity) and, after that, the discretization error can be controlled by decreasing the step $d$ of the grid in $x$-space, equivalently, increasing the number of points $M=2^{m}$ of the grid. Now, consider the inverse Fourier transform. Assuming that the truncated region on the line $\operatorname{Im} \xi=\omega$ is of the form $[-\Lambda+i \omega, \Lambda+i \omega]$, and denoting the step of the uniform grid by $\zeta$, we have $d \zeta /(2 \pi)=1 / M$, hence, $\Lambda=2^{m-1} \zeta=\pi / d$. It follows that if we keep the truncated region in $x$-space fixed and decrease $d$ (equivalently, increase $m$ ) then we can control the truncation error of iFFT but not the discretization error. To control the latter, we need to increase the truncated region in $x$-space and, in addition, increase $M$ by a larger factor. In the numerical examples in Section [5] we will use the truncated region in $x$-space sufficiently large so that the doubling the region and increasing the number of points 4 -fold changes the option price by $0.01 \%$ or less.

c) In N.Boyarchenko and Levendorskii (2007), it was shown that the discretization error for iFFT can be made smaller by an appropriate choice of the line of the integration. As a rule of thumb, $\omega=\lambda_{+} / 3$ for positive $\omega$ was suggested; the natural analog for negative $\omega$ is $\lambda_{-} / 3$.

\section{Approximation of factors in the Wiener-Hopf factorization formula}

We consider three procedures for approximations of the Wiener-Hopf factors; the list can be extended. The first two procedures should be used with an appropriate choice of the line of integration described above. The third procedure is not so straightforward but it involves only real $\xi$ and uses the real Fourier transform, which is faster than the standard (complex) Fourier transform. 


\subsection{Realizations of general formulas for $\phi_{q}^{ \pm}(\xi)$ using FFT and iFFT}

The first two approximations are straightforward realizations of explicit general formulas for the Wiener-Hopf factors. If $q>0$ is sufficiently large (equivalently, the time-step sufficiently small), then $\operatorname{Re}(q+\psi(\xi))>0$ for all $\xi$ in the strip $\operatorname{Im} \xi \in\left(\lambda_{-}, \lambda_{+}\right)$. Take a negative $\omega_{-}>\lambda_{-}$. Boyarchenko and Levendorskii (see eq. (3.58) and (3.60) in Boyarchenko and Levendorskii (2002b)) proved that for $\xi$ in the upper half-plane $\operatorname{Im} \xi>0$,

$$
\phi_{q}^{+}(\xi)=\exp \left[\frac{1}{2 \pi i} \int_{\operatorname{Im} \eta=\omega_{-}} \frac{\xi \ln (q+\psi(\eta))}{\eta(\xi-\eta)} d \eta\right],
$$

for any $\omega_{-} \in\left(\lambda_{-}, 0\right)$, and for any $\xi$ in the lower half-plane $\operatorname{Im} \xi<0$,

$$
\phi_{q}^{-}(\xi)=\exp \left[-\frac{1}{2 \pi i} \int_{\operatorname{Im} \eta=\omega_{+}} \frac{\xi \ln (q+\psi(\eta))}{\eta(\xi-\eta)} d \eta\right],
$$

where $\omega_{+} \in\left(0, \lambda_{+}\right)$. In the case of a put option, we use (46) to calculate $\phi_{q}^{+}(\xi)$ on the line $\operatorname{Im} \xi=\omega>0$, and then calculate $\phi_{q}^{-}(\xi)=q /\left((q+\psi(\xi)) \phi_{q}^{+}(\xi)\right)$. In the case of a call option, we use (46) to calculate $\phi_{q}^{-}(\xi)$ on the line $\operatorname{Im} \xi=\omega<-1$, and then calculate $\phi_{q}^{+}(\xi)=q /\left((q+\psi(\xi)) \phi_{q}^{-}(\xi)\right)$.

The integrals under the exponential sign needs to be calculated at the points of the chosen grid on the line $\operatorname{Im} \xi=\omega$. This can be done using FFT and iFFT. For instance, for $\operatorname{Im} \xi=\omega>0$ and $\omega_{-} \in\left(\lambda_{-}, 0\right)$,

$$
\frac{1}{2 \pi i} \int_{\operatorname{Im} \eta=\omega_{-}} \frac{\xi \ln (q+\psi(\eta))}{\eta(\xi-\eta)} d \eta=\xi F_{x \rightarrow \xi} \mathbf{1}_{(-\infty, 0]}(x) F_{\eta \rightarrow x} \frac{\ln (q+\psi(\eta))}{i \eta},
$$

and for $\operatorname{Im} \xi=\omega<0$ and $\omega_{+} \in\left(0, \lambda_{+}\right)$,

$$
\frac{1}{2 \pi i} \int_{\operatorname{Im} \eta=\omega_{+}} \frac{\xi \ln (q+\psi(\eta))}{\eta(\xi-\eta)} d \eta=\xi F_{x \rightarrow \xi} \mathbf{1}_{[0,+\infty)}(x) F_{\eta \rightarrow x} \frac{\ln (q+\psi(\eta))}{i \eta} .
$$

There exists a similar albeit more involved pair of explicit formulas for the Wiener-Hopf factors (see Section 3.6.2 in Boyarchenko and Levendorskii $(2002 \mathrm{~b})$ ), with a better rate of convergence of the integrals. This pair of formulas also can be realized using the FFT and iFFT. The new ingredient is the reduction of the factorization problems to symbols of order 0 , which stabilize at infinity to 1 . We explain the reduction for processes of order $\nu \in(1,2]$ and of order $\nu \in(0,1]$, without drift. Introduce functions

$$
\begin{aligned}
\Lambda_{-}(\xi) & =\lambda_{+}^{\nu / 2}\left(\lambda_{+}+i \xi\right)^{-\nu / 2} \\
\Lambda_{+}(\xi) & =\left(-\lambda_{-}\right)^{\nu / 2}\left(-\lambda_{-}-i \xi\right)^{-\nu / 2} \\
\Phi(\xi) & =q\left((q+\phi(\xi)) \Lambda_{+}(\xi) \Lambda_{-}(\xi)\right)^{-1}
\end{aligned}
$$

Functions $\Lambda_{ \pm}(\xi)$ are analytic and do not vanish in the half-plane $\pm \operatorname{Im} \xi>0$, continuous up to the boundary. In addition, $\Lambda_{ \pm}(\xi)$ and its reciprocal grow not faster than a polynomial. Therefore, it remains to factorize

$$
\Phi(\xi)=\Phi^{+}(\xi) \Phi^{-}(\xi),
$$


and then set

$$
\phi_{q}^{ \pm}(\xi)=\Lambda_{ \pm}(\xi) \Phi^{ \pm}(\xi)
$$

Functions $\Phi^{ \pm}$can be calculated using explicit formulas

$$
\begin{aligned}
\Phi^{+}(\xi) & =\exp \left[\frac{1}{2 \pi i} \int_{\operatorname{Im} \eta=\omega_{-}} \frac{\ln \Phi(\eta)}{\xi-\eta} d \eta\right], \\
\Phi^{-}(\xi) & =\exp \left[-\frac{1}{2 \pi i} \int_{\operatorname{Im} \eta=\omega_{+}} \frac{\ln \Phi(\eta)}{\xi-\eta} d \eta\right] .
\end{aligned}
$$

For model classes of RLPEs, $\log \Phi(\eta)$ decays at infinity as $|\eta|^{-1}$, therefore the integrals under the exponential signs can be calculated efficiently.

\subsection{Approximation of symbols}

The last and computationally most efficient realization of the Wiener-Hopf method obtains as we, first, approximate $\Phi$ by a periodic function $\Phi_{d}$ with a large period $2 \pi / d$, which is the length of the truncated region in $\xi$-state, then approximate the latter by a partial sum of the Fourier series, and, finally, use the factorization of the latter instead of the exact one. The second step is straightforward. We impose an additional condition

$$
\left|\Phi^{\prime}(\xi)\right| \leq C(1+|\xi|)^{-\rho}
$$

where $\rho>0$; this condition is satisfied by all RLPEs (and can be relaxed), which makes the following lemma applicable.

Consider a function $f_{d} \in C^{1}[-\pi / d, \pi / d]$ depending on a small parameter $d$, and, for $m>0$, construct the partial sum

$$
S_{m}\left(f_{d}\right)=\frac{d}{2 \pi} \sum_{|k| \leq m} \hat{f}_{d, k} e^{i d k x}
$$

of the Fourier series for $f_{d}$.

Lemma 4.1. Let $f_{d}(-\pi / d)=f_{d}(\pi / d)$, and let there exists $C>0$ such that for all $x \in[-\pi / d, \pi / d]$ and all $d \in(0,1],\left|\partial_{x} f_{d}(x)\right| \leq C$.

Then there exists a function $d \mapsto m_{0}(d)$ such that $\forall m \geq m_{0}(d), x \in$ $[-\pi / d, \pi / d]$ and $d \in(0,1]$,

$$
\left|f_{d}(x)-S_{m}\left(f_{d}\right)(x)\right| \leq \epsilon .
$$

Proof. Changing the variables $x=x^{\prime} / d$, we see that it suffices to prove lemma for a $g_{d} \in C^{1}[-\pi, \pi]$, such that $g_{d}(-\pi)=g_{d}(\pi)$ and for all $x \in[-\pi, \pi]$ and all $d \in(0,1],\left|\partial_{x} g_{d}(x)\right| \leq C / d$. Set

$$
S_{m}\left(d_{d}\right)=\frac{1}{2 \pi} \sum_{|k| \leq m} \hat{g}_{d, k} e^{i k x} .
$$

We need to prove that there exists a function $d \mapsto m_{0}(d)$ such that $\forall m \geq m_{0}(d)$, $x \in[-\pi, \pi]$ and $d \in(0,1]$,

$$
\left|g_{d}(x)-S_{m}\left(g_{d}\right)(x)\right| \leq \epsilon .
$$


The proof is a modification of the classical proof of the uniform convergence of the Fourier series due to U.Dini. We use

$$
S_{n}\left(g_{d}\right)(x)-g_{d}(x)=\frac{1}{\pi} \int_{0}^{\pi} h_{d}(x, t) \frac{\sin ((n+1 / 2) t)}{2 \sin (t / 2)} d t
$$

where $h_{d}(x, t)=g_{d}(x+t)+g_{d}(x-t)-2 g_{d}(x)$, which presumes that $g_{d}$ is extended to a periodic function. For any $\epsilon>0$, set $\delta=\epsilon d /(2 C)$. Then

$$
\left|\frac{1}{\pi} \int_{0}^{\delta} h_{d}(x, t) \frac{\sin ((n+1 / 2) t)}{2 \sin (t / 2)} d t\right| \leq \frac{1}{2} \int_{0}^{\delta} \frac{\left|h_{d}(x, t)\right|}{t} d t \leq \delta C / d \leq \epsilon / 2 .
$$

Next, integrating by parts,

$$
\begin{aligned}
\left|\frac{1}{\pi} \int_{\delta}^{\pi} h_{d}(x, t) \frac{\sin ((n+1 / 2) t)}{2 \sin (t / 2)} d t\right| & \leq \frac{1}{2 \delta(n+1 / 2)} \mid \cos ((n+1 / 2) t) h_{d}(x, t)_{t=\delta}^{t=\pi} \\
& -\int_{\delta}^{\pi} \cos ((n+1 / 2) t) \partial_{t} h_{d}(x, t) d t \mid \leq \frac{A(d)}{n+1 / 2},
\end{aligned}
$$

where $A(d)$ depends on $d$ and $C$ but not on $n$. Choosing $m_{0}>2 A(d) / \epsilon$ and taking (58) into account, we obtain (57).

The first and third steps are more subtle. Essentially, we want to approximate the bounded continuous solution of the problem

$$
\begin{aligned}
\Phi(D) u(x) & =f(x), \quad x>0, \\
u(x) & =0,
\end{aligned}
$$

with the bounded continuous solution of the problem

$$
\begin{aligned}
\Phi_{d}(D) u_{d}(x) & =f(x), \quad x>0, \\
u_{d}(x) & =0 .
\end{aligned}
$$

By the Sobolev embedding theorem, $C \subset H^{1 / 2+\gamma}$ for any $\gamma>0$. It follows that, for our purposes, it suffices to prove an estimate of the form $\left\|u-u_{d}\right\|_{1 / 2+\gamma} \rightarrow 0$ as $d \rightarrow 0$, for some $\gamma>0$. Certainly, this statement cannot be valid for an arbitrary $f$. However, each time when we need to solve a boundary problem of the form (59)- 60), function $f$ is more regular than just continuous. Indeed, outside the barrier and strike, it is of the class $H^{p}$ for any $p$, near the barrier it is of the class $H^{1 / 2+\nu / 2-\gamma}$ for any $\gamma>0$, and near the strike, it is of the class $H^{3 / 2-\gamma}$ for any $\gamma>0$ at the first step of the backward induction. At each next step, the regularity in a neighborhood of the strike increases - for more details, see Levendorskii (2004b). This means that outside an arbitrarily small neighborhood of the barrier and strike, $\mathbf{1}_{|\cdot| \geq \pi / d}(D)\left(u-u_{d}\right)$ vanishes very fast in $H^{p}$-norm, for any $p>0$, as $d \rightarrow+\infty$. In a small neighborhood of the barrier, we can guarantee only some rate of decay of the function $\mathbf{1}_{|\cdot| \geq \pi / d}(D)\left(u-u_{d}\right)$ in $H^{1 / 2+\nu / 2-\gamma}$-norm, for arbitrary small $\gamma>0$ (the constant in the estimate for the norm depends on $\gamma$ ). In a small vicinity of the strike, the error is small in $H^{3 / 2+\nu / 2-\gamma}$-norm, therefore, the rate of decay in $H^{1 / 2+\nu / 2-\gamma}$-norm will be much better. Should the estimate near the barrier be global, the convergence of the method would had been poor. However, the symbol $\psi$ and the factors $\phi^{ \pm}$are 
analytic in a strip that contains the real line, and the strip is, typically, rather wide. This means that relative large errors localized in a small neighborhood of the barrier and strike will remain localized at the next steps of the backward induction procedure, and we may hope that the resulting errors will be small outside a small vicinity of the barrier - but not very small in a neighborhood of the strike. The numerical examples in Section [5 support this claim.

Note that the schematic argument above can be made precise. This is an example of a rather simple albeit tedious application of microanalytical considerations - see, e.g., Eskin (1973) and Hörmander (1985).

\subsection{Explicit formulas for approximations of $\phi^{ \pm}$}

For small positive $d$ and large even $M$ (according to Lemma 4.1] $M$ should increase faster than $1 / d)$, set

$$
\begin{aligned}
b_{k}^{d} & =\frac{d}{2 \pi} \int_{-\pi / d}^{\pi / d} \ln \Phi(\xi) e^{-i \xi k d} d \xi, \quad k \neq 0, \\
b_{d, M}(\xi) & =\sum_{k=-M / 2+1}^{M / 2} b_{k}^{d}(\exp (i \xi k d)-1), \\
b_{d, M}^{+}(\xi) & =\sum_{k=1}^{M / 2} b_{k}^{d}(\exp (i \xi k d)-1), \\
b_{d, M}^{-}(\xi) & =\sum_{k=-M / 2+1}^{-1} b_{k}^{d}(\exp (i \xi k d)-1) \\
\Phi_{d, M}(\xi) & =\exp \left(b_{d, M}(\xi)\right), \\
\Phi_{d, M}^{ \pm}(\xi) & =\exp \left(b_{d, M}^{ \pm}(\xi)\right) .
\end{aligned}
$$

\subsection{Approximation of $\phi_{q}^{ \pm}(D)$ using Fast Fourier Transform}

Let $d$ be the step in $x$-space, $\zeta$-the step in $\xi$-space, and $M=2^{m}$ the number of the points on the grid; decreasing $d$ and increasing (even faster) $M$, we obtain a sequence of approximations to the option price.

Approximants for EPV-operators can be efficiently computed by using the Fast Fourier Transform (FFT). Consider the algorithm (the discrete Fourier transform (DFT)) defined by

$$
G_{l}=\operatorname{DFT}[g](l):=\sum_{k=0}^{M-1} g_{k} e^{2 \pi i k l / M}, \quad l=0, \ldots, M-1 .
$$

(It differs in sign in front of $i$ from the algorithm fft in MATLAB). The DFT maps $m$ complex numbers (the $g_{k}$ 's) into $m$ complex numbers (the $G_{l}$ 's). The formula for the inverse DFT which recovers the set of $g_{k}$ 's exactly from $G_{l}$ 's is:

$$
g_{k}=i \operatorname{DFT}[G](k):=\frac{1}{M} \sum_{l=0}^{M-1} G_{l} e^{-2 \pi i k l / M}, \quad k=0, \ldots, M-1 .
$$

In our case, the data consist of a real-valued array $\left\{g_{k}\right\}_{k=0}^{M}$. The resulting transform satisfies $G_{M-l}=\bar{G}_{l}$. Since this complex-valued array has real values 
$G_{0}$ and $G_{M / 2}$, and $M / 2-1$ other independent complex values $G_{1}, \ldots, G_{M / 2-1}$, then it has the same "degrees of freedom" as the original real data set. In this case, it is inefficient to use full complex FFT algorithm. The main idea of FFT of real functions is to pack the real input array cleverly, without extra zeros, into a complex array of half of length. Then a complex FFT can be applied to this shorter length; the trick is then to get the required values from this result (see Press, W. et al (1992) for details). To distinguish DFT of real functions we will use notation RDFT. Formulas (69)-(70) turn into:

$$
\begin{aligned}
G_{l}=\operatorname{RDFT}[g](l) & :=\sum_{k=0}^{M-1} g_{k} e^{2 \pi i k l / M}, \quad l=0, \ldots, M / 2 ; \\
g_{k}=i \operatorname{RDFT}[G](k) & :=\frac{2}{M} \operatorname{Re} \sum_{l=1}^{M / 2-1} G_{l} e^{-2 \pi i k l / M}+\frac{1}{M}\left(G_{0}+G_{M / 2} e^{-i \pi k j 2}\right)
\end{aligned}
$$

$k=0, \ldots, M-1$. Fix the space step $d>0$ and number of the space points $M=2^{m}$. Define the partitions of normalized log-price domain $\left[-\frac{M d}{2} ; \frac{M d}{2}\right)$ by points $x_{k}=-\frac{M d}{2}+k d, k=0, \ldots, m-1$, and frequency domain $\left[-\frac{\pi}{d} ; \frac{\pi}{d}\right]$ by points $\xi_{l}=\frac{2 \pi l}{d M}, l=-M / 2, \ldots, M / 2$. Then the Fourier transform of a function $g$ on the real line can be approximated as follows:

$$
\hat{g}\left(\xi_{l}\right) \approx \int_{-M d / 2}^{M d / 2} e^{-i x \xi_{l}} g(x) d x \approx \sum_{k=0}^{M-1} g\left(x_{k}\right) e^{-i x_{k} \xi_{l}} d=d e^{i \pi l} \sum_{k=0}^{M-1} g\left(x_{k}\right) e^{-2 \pi i k l / M},
$$

and finally,

$$
\hat{g}\left(\xi_{l}\right) \approx d e^{i \pi l} \overline{R D F T[g](l)}, \quad l=0, \ldots, M / 2 .
$$

Here $\bar{z}$ denotes the complex conjugate of $z$. Now, we approximate $\mathcal{E}$. Using the notation $p(\xi):=q(q+\psi(\xi))^{-1}$, we have

$$
\begin{aligned}
(\mathcal{E} g)\left(x_{k}\right)= & \frac{1}{2 \pi} \int_{-\infty}^{\infty} e^{i x_{k} \xi} p(\xi) \hat{g}(\xi) d \xi \\
\approx & \frac{1}{2 \pi} \sum_{l=-M / 2+1}^{M / 2} e^{-i x_{k} \xi_{l}} p\left(-\xi_{l}\right) \overline{\hat{g}\left(\xi_{l}\right)} \frac{2 \pi}{d M} \\
\approx & \frac{2}{M} \operatorname{Re} \sum_{l=1}^{M / 2-1} e^{-2 \pi i k l / M} p\left(-\xi_{l}\right) \operatorname{RDFT}[g]_{l} \\
& +\frac{1}{M}\left(\operatorname{RDFT}[g]_{0}+\operatorname{Re} p\left(-\xi_{M / 2}\right) \operatorname{RDFT}[g]_{M / 2}\right)
\end{aligned}
$$

Finally,

$$
(\mathcal{E} g)\left(x_{k}\right) \approx i \operatorname{RDFT}[\bar{p} . * \operatorname{RDFT}[g]](k), \quad k=0, \ldots, M-1 .
$$

Next, we approximate operators $\mathcal{E}^{ \pm}=\phi_{q}^{ \pm}(D)$. First, we find an approximation of function $\ln \Phi$ by the Fourier series using the formula (64). Note that the coefficients $b_{k}^{d}$ in (64) are defined by (63) and can be efficiently computed by 
using iRDFT. We have:

$$
\begin{aligned}
b_{k}^{d} & =\frac{d}{2 \pi} \int_{-\pi / d}^{\pi / d} \ln \Phi(\xi) e^{-i \xi k d} d \xi \\
& \approx \frac{d}{2 \pi} \sum_{l=-M / 2+1}^{M / 2} \ln \Phi\left(\xi_{l}\right) e^{-2 \pi i k l / M} \frac{2 \pi}{M d} \\
& =i \operatorname{RDFT}[\ln \Phi](k) .
\end{aligned}
$$

Then we calculate $b_{d, M}^{ \pm}$in (65)- 66), and, finally, calculate the approximations to the Wiener-Hopf factors

$$
p^{ \pm}\left(\xi_{l}\right)=\Lambda_{-\nu / 2}^{ \pm}\left(\xi_{l}\right) \exp \left(b_{d, M}^{ \pm}\left(\xi_{l}\right)\right), l=-M / 2, \ldots, 0 .
$$

The action of the EPV-operator $\mathcal{E}^{ \pm}$is approximated as follows:

$$
\begin{aligned}
\left(\mathcal{E}^{ \pm} g\right)\left(x_{k}\right) & =\frac{1}{2 \pi} \int_{-\infty}^{\infty} e^{i x_{k} \xi} \phi^{ \pm}(\xi) \hat{g}(\xi) d \xi \\
& \approx \frac{1}{2 \pi} \int_{-\pi / d}^{\pi / d} e^{-i x_{k} \xi} p^{ \pm}(-\xi) \overline{\hat{g}(\xi)} d \xi \\
& \approx \frac{1}{2 \pi} \sum_{l=-M / 2+1}^{M / 2} e^{-i x_{k} \xi_{l}} p^{ \pm}\left(-\xi_{l}\right) \overline{\hat{g}\left(\xi_{l}\right)} \frac{2 \pi}{d M}
\end{aligned}
$$

and, finally,

$$
\left(\mathcal{E}^{ \pm} g\right)\left(x_{k}\right)=i \operatorname{RDFT}\left[\overline{p^{ \pm}} \cdot \operatorname{RDFT}[g]\right](k), \quad k=0, \ldots, M-1 .
$$

\subsection{Algorithm of the FWH-method}

We assume that the "drift" $\mu=0$; non-zero $\mu$ can be eliminated by the change of variables $x=x^{\prime}+\mu t$.

I. Preliminary STEPS

Step 1. Input $r$ and parameters of the characteristic exponent $\psi(\xi)$ (see (11)(12) $)$.

Step 2. Input space step $d$.

Step 3. Input $x_{\min }$ and $x_{\max }$ - the lower and upper bounds for the space variable $x$. The choice $x_{\min }=\ln (0.4)+\min \{\ln H, \ln K\}$ and $x_{\max }=$ $\ln (2.5)+\max \{\ln H, \ln K\}$ is optimal for typical parameter values.

Step 4. Define the number of space points $M$ as follows. Find a positive integer $m$ such that $2^{m-1}<\frac{x_{\max }-x_{\min }}{2 d} \leq 2^{m}$, and set $M=2^{m}$.

Step 5. Input time to matirity $T$, the number of time steps $N$, and set $\Delta t=$ $T / n, q=(\Delta t)^{-1}+r$.

Step 6. Set $\xi_{k}=\frac{\pi k}{M d}, k=-M+1, \ldots, M$, and find $p_{k}=q\left(q+\psi\left(\xi_{k}\right)\right)^{-1}, k=$ $-M+1, \ldots,-1$, and $p_{0}=1, p_{-M}=q \operatorname{Re}\left(q+\psi\left(-\xi_{M}\right)\right)^{-1}$. We need array $p_{k}, k=-M, \ldots, 0$ for approximation of $\mathcal{E}$ (see (74)). 
Step 7. Find $(\ln \Phi)_{k}=\ln \Phi\left(\xi_{k}\right), k=1, \ldots, M-1$, and $(\ln \Phi)_{0}=0,(\ln \Phi)_{M}=$ $\operatorname{Re} \ln \Phi\left(\xi_{M}\right)$. We need array $(\ln \Phi)_{k}, k=-M, \ldots, 0$ for calculation of coefficients $b_{k}^{d}$ (see (64) and (75)).

Step 8. Using inverse FFT for real-valued functions, we find (see (75))

$$
b_{k}:=b_{k}^{d}=i R D F T[\ln \Phi](k), \quad k=-M+1, \ldots, M
$$

Step 9. Set $b_{0}^{-}=-\sum_{l=-M+1}^{l=-1} b_{l}, b_{k}^{-}=b_{-k}, k=1, \ldots, M b_{k}^{-}=0, k=-M+$ $1, \ldots,-1$. We need the array $b_{k}^{-}, k=-M+1, \ldots, M$ for calculation of $b^{-}\left(-\xi_{l}\right)$ (see (66), (76) and (777)

Step 10. Find $b^{-}\left(-\xi_{l}\right)=R D F T\left[b^{-}\right](l), l=0, \ldots, M$.

Step 11. Set $b_{0}^{+}=b_{0}-b_{0}^{-}, b_{k}^{+}=b_{-k}, k=-M+1, \ldots,-1 b_{k}^{+}=0, k=1, \ldots, M$. We need array $b_{k}^{+}, k=-M+1, \ldots, M$ for calculation of $b^{+}\left(-\xi_{l}\right)$ (see (65), (76) and (77))

Step 12. Calculate $b^{+}\left(-\xi_{l}\right)=R D F T\left[b^{+}\right](l), l=0, \ldots, M$.

Step 13. We find $p_{l}^{ \pm}=\Lambda_{ \pm}\left(\xi_{l}\right) \exp \left(b^{ \pm}\left(\xi_{l}\right)\right), l=-M, \ldots, 0$ (see (76) $)$. We need arrays $p_{l}^{ \pm}, l=-M, \ldots, 0$ for the approximation of $\mathcal{E}^{ \pm}$(see (77)).

II PRICING BARRIER OPTION

Denote $v_{j}^{k}=v_{j}\left(x_{k}\right)$ and $w_{j}^{k}=w_{j}\left(x_{k}\right), k=-M+1, \ldots, M, j=N, N-1, \ldots, 0$.

Step 1. Input barrier $H$. Set $\gamma=(q \Delta t)^{-1}$.

Step 2. Calculate values of payoff function: $v_{N}^{k}=G\left(H e^{x_{k}}\right), k=-M+1, \ldots, M$.

Step 3. Calculate (see (42) and (77))

$$
w_{N-1}^{k}=i R D F T\left[\overline{p^{+}} \cdot R D F T\left[v_{N}\right]\right](k), \quad k=-M+1, \ldots, M .
$$

Step 4. Calculation in cycle $s=N-1, N-2, \ldots, 1$.

a. Set $w_{s}^{k}=0, k \leq 0$.

b. Applying direct and inverse FFT, we calculate (see (44) and (74))

$$
w_{s-1}^{k}=\gamma i \operatorname{RDFT}\left[\bar{p} \cdot \operatorname{RDFT}\left[w_{s}\right]\right](k), k=-M+1, \ldots, M
$$

Step 5. Set $w_{0}^{k}=0, k \leq 0$.

Step 6. Applying direct and inverse FFT, we calculate (see (43) and (77))

$$
v_{0}^{k}=\gamma \operatorname{RiRFT}\left[\overline{p^{-}} \cdot \operatorname{RDFT}\left[w_{0}\right]\right](k), \quad k=-M+1, \ldots, M
$$

Step 7. Output: spot price: $S_{k}=H e^{x_{k}}$; option price: $V_{k}=v_{0}^{k}, k=1, \ldots, M$. 


\section{$5 \quad$ Numerical Examples}

In this section, we compare the performance of the three methods: $\mathrm{CV}$-method, FWH-method and IWH-method. We consider the down-and-out put option with strike $K$, barrier $H$ and time to expiry $T$. The option prices were calculated on a PC with characteristics Intel Core(TM)2 Due CPU, 1.8GHz, RAM 1024Mb, under Windows Vista. For computation of the prices by $\mathrm{CV}$-method we used Premia 8 routine. We consider two types of processes, three times to maturity, and two ratios $H / K$. Certainly, it would be interesting to study more variants but a detailed study of several case will require dozens of tables. The examples, which we analyze in detail below, are fairly representative. The option price depends on several parameters of the chosen scheme. For a fixed number of time steps, $N$, and step in $x$-space, $d$, we will vary the other parameters of the scheme to ensure that the price does not change significantly (the details will be explained below). This explains why we can denote the price by $V_{d, N}$ (the scheme will be indicated separately).

\subsection{Processes of order $\nu<1$}

For processes of order $\nu<1$, we use FDS-method as the benchmark. The reason is that it is more accurate than CV-method albeit slower. The approximation error (of small jumps) of FDS-method is smaller than that of CV-method, and the discretization errors are, essentially, of the same nature. In addition, at each time step, FDS-method is an iterative method, whereas CV-method is an explicit-implicit one, which makes the former more accurate than the latter. To sum up, one should expect that $\mathrm{CV}$-method produces larger errors than FDS method. We run FDS choosing very fine grids in both time and state space, and sufficiently large truncated domain in $x$-space. Sufficiently large means that, as we increase the truncated region and number of points on the grid two-fold, the option prices change by $0.01 \%$ or less.

We take KoBoL model with parameters $\sigma=0, \nu=0.5, \lambda_{+}=9, \lambda_{-}=-8$, $c=1$. We choose instantaneous interest rate $r=0.072310$, time to expiry $T=0.5$ year, strike price $K=100$ and the barrier $H=90$. In this case, the drift parameter $\mu$ is approximately zero. The localization domain is $\left(x_{\min } ; x_{\max }\right)$ with $x_{\min }=-\ln 2$ and $x_{\max }=\ln 2$; we check separately that if we increase the domain two-fold, and the number of points 4-fold, the prices change by less than 0.0001. In Table 5.1, we show option prices calculated with FDS, FWH and CV methods, with very fine grids, and relative errors w.r.t. FDS. ExtFWH (resp., ExtCV) labels option prices obtained by linear extrapolation of prices $V_{d, N}$ with $d=0.00025$ and $d=0.0001$ (resp., $d=0.000005$ and $d=0.000002$ ). FDS-

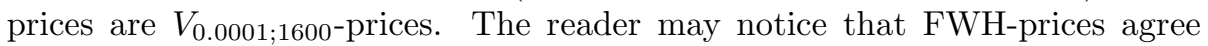
with FDS-prices very well (relative error less than $1 \%$ even in the out-of-the money region) after $5 \mathrm{sec}$ whereas $\mathrm{CV}$-method produces relative errors larger than $3 \%$ after dozens of hours of calculation. In Table 5.2, we see that FWH produces good results in just 1-2 seconds. Table 5.3 shows prices $V_{d, N}$ and errors of CV-method for several pairs $(d, N)$. Finally, in Table 5.4, we demonstrate that the relative differences between prices obtained with FWH-method and IWH-methods are very small, and that the former method is much faster than the latter. 
Table 1: Down-and-out put prices in KoBoL model, $\nu=0.5$

\begin{tabular}{|l|c|c|c|c|c|c|c|}
\hline & FDS & \multicolumn{3}{|c|}{ FWH } & \multicolumn{3}{c|}{ CV } \\
\hline Spot price & & $d=0.00025$ & $d=0.0001$ & ExtFWH & $d=0.000005$ & $d=0.000002$ & ExtCV \\
\hline$S=91$ & 0.2359 & 0.2340 & 0.2350 & 0.2357 & 0.2186 & 0.2236 & 0.2269 \\
$S=101$ & 0.5669 & 0.5657 & 0.5666 & 0.5671 & 0.5522 & 0.5567 & 0.5598 \\
$S=111$ & 0.3850 & 0.3847 & 0.3852 & 0.3855 & 0.3775 & 0.3800 & 0.3816 \\
$S=121$ & 0.2081 & 0.2080 & 0.2083 & 0.2084 & 0.2046 & 0.2057 & 0.2065 \\
$S=131$ & 0.1073 & 0.1073 & 0.1074 & 0.1075 & 0.1055 & 0.1061 & 0.1065 \\
\hline CPU-time(sec) & 97,300 & 5 & 12 & & 26,000 & 116,000 & \\
\hline
\end{tabular}

Relative errors

\begin{tabular}{|l|c|c|c|c|c|c|c|}
\hline & FDS & \multicolumn{3}{|c|}{ FWH } & \multicolumn{3}{c|}{ CV } \\
\hline Spot price & & $d=0.00025$ & $d=0.0001$ & ExtFWH & $d=0.000005$ & $d=0.000002$ & ExtCV \\
\hline$S=91$ & 0.235866 & -0.0079 & -0.0035 & -0.0005 & -0.0731 & -0.0520 & -0.0379 \\
$S=101$ & 0.566907 & -0.0021 & -0.0005 & 0.0006 & -0.0260 & -0.0179 & -0.0125 \\
$S=111$ & 0.384982 & -0.0008 & 0.0005 & 0.0014 & -0.0195 & -0.0130 & -0.0087 \\
$S=121$ & 0.208093 & -0.0004 & 0.0008 & 0.0017 & -0.0175 & -0.0115 & -0.0075 \\
$S=131$ & 0.107307 & -0.0004 & 0.0008 & 0.0017 & -0.0168 & -0.0111 & -0.0073 \\
\hline
\end{tabular}

KoBoL parameters: $\nu=0.5, \lambda_{+}=9, \lambda_{-}=-8, c=1$.

Option parameters: $K=100, H=90, r=0.072310, T=0.5$.

Algorithm parameters: $d$ - space step, $N=1600$ - number of time steps, $S-$ spot price.

Table 2: Down-and-out put prices in KoBoL model, FWH-method

\begin{tabular}{|l|c|c|c||c|c|c|}
\hline \multicolumn{1}{|c|}{} & \multicolumn{3}{c||}{ Option price, $V_{0.000, N}(S)$} & \multicolumn{3}{c|}{ Relative errors w.r.t. FDS } \\
\hline Spot price & $N=50$ & $N=100$ & $N=200$ & $N=50$ & $N=100$ & $N=200$ \\
\hline$S=91$ & 0.2424 & 0.2371 & 0.2345 & 0.0278 & 0.0053 & -0.0057 \\
$S=101$ & 0.5723 & 0.5681 & 0.5660 & 0.0095 & 0.0022 & -0.0015 \\
$S=111$ & 0.3806 & 0.3826 & 0.3831 & -0.0114 & -0.0071 & -0.0049 \\
$S=121$ & 0.2048 & 0.2062 & 0.2069 & -0.0157 & -0.0090 & -0.0055 \\
$S=131$ & 0.1059 & 0.1065 & 0.1068 & -0.0133 & -0.0077 & -0.0049 \\
\hline CPU-time(sec) & 0.05 & 0.1 & 0.2 & & & \\
\hline
\end{tabular}

KoBoL parameters: $\nu=0.5, \lambda_{+}=9, \lambda_{-}=-8, c=1$.

Option parameters: $K=100, H=90, r=0.072310, T=0.5$.

Algorithm parameters: $d=5 \cdot 10^{-4}$ - space step, $N$ - number of time steps, $S$ - spot price.

Then we consider the same model, but for smaller time to expiry $T=0.1$ year. Tables 5.5-5.7 confirm the observations described above.

\subsection{Processes of order $\nu=1$. NIG as an example}

For processes of order $\nu \geq 1$, we do not have a clear favorite. The following argument justifies the use of FWH-method. First, as we saw above, for processes of order $\nu<1$, FWH is in a good agreement with the benchmark (and in extremely good agreement with IWH). If the truncated region in $x$-space is wide enough, then any further change of its size is, essentially, irrelevant because of the exponential decay of the jump density. This argument works only when we make approximations in $x$-space. Since IWH-method and FWH-method 
Table 3: Down-and-out put prices in KoBoL model, CV-method

\begin{tabular}{|c|c|c|c|c|c|c|c|c|c|c|c|c|}
\hline & \multicolumn{12}{|c|}{ Option price, $V_{d, N}(S)$} \\
\hline$d$ & $\begin{array}{c}10^{-3} \\
1600\end{array}$ & $\begin{array}{l}5 \cdot 10^{-4} \\
1600\end{array}$ & $2.5 \cdot 10^{-4}$ & $\begin{array}{c}10^{-4} \\
1600\end{array}$ & $\begin{array}{c}5 \cdot 10^{-5} \\
1600\end{array}$ & \multicolumn{2}{|c|}{$2.5 \cdot 10^{-5}$} & \multicolumn{2}{|c|}{$10^{-5}$} & \multicolumn{2}{|c|}{$5 \cdot 10^{-6}$} & $\begin{array}{c}2 \cdot 10^{-6} \\
18000\end{array}$ \\
\hline $\begin{array}{c}N \\
S=91\end{array}$ & $\frac{1600}{0.092168}$ & $\frac{1600}{0.111911}$ & $\frac{1600}{0.134079}$ & $\frac{1600}{0.163195}$ & 0.183077 & \multicolumn{2}{|l|}{$\frac{.1900269}{0.1992}$} & \multicolumn{2}{|c|}{6400} & \multicolumn{2}{|c|}{10000} & $\frac{18000}{0.223599}$ \\
\hline$S=101$ & 0.448530 & 0.474713 & 0.496496 & 0.163195 & 0.530187 & \multicolumn{2}{|c|}{0.538883} & \multicolumn{2}{|c|}{$\begin{array}{l}0.212927 \\
0.547436\end{array}$} & \multicolumn{2}{|c|}{0.218617} & \\
\hline$S=111$ & 0.324012 & 0.336316 & 0.347433 & $\begin{array}{l}0.517848 \\
0.358812\end{array}$ & 0.365830 & \multicolumn{2}{|l|}{0.370336} & & & \\
\hline$S=121$ & 0.177738 & 0.184006 & 0.189574 & & \multirow{2}{*}{$\begin{array}{l}0.198729 \\
0.102641\end{array}$} & \multicolumn{2}{|c|}{0.200939} & & \multicolumn{2}{|c|}{0.377460} & \\
\hline$S=131$ & 0.092162 & 0.095352 & 0.098134 & $\begin{array}{l}0.195227 \\
0.100932\end{array}$ & & 0.103747 & & \multicolumn{2}{|c|}{$\begin{array}{l}0.203187 \\
0.104868\end{array}$} & \multicolumn{2}{|c|}{0.105499} & $\begin{array}{l}0.379963 \\
0.205700\end{array}$ \\
\hline CPU-time (sec) & 7.5 & 15 & 35 & 77 & 420 & 1950 & & 110 & & & & 116000 \\
\hline & & & & Relative & errors w.r.t. & FDS & & & & & & \\
\hline $\begin{array}{l}d \\
N\end{array}$ & $\begin{array}{l}10^{-3} \\
1600\end{array}$ & $\begin{array}{c}5 \cdot 10^{-4} \\
1600\end{array}$ & $\begin{array}{r}2.5 \cdot 10^{-} \\
1600\end{array}$ & $\begin{array}{l}10^{-4} \\
1600\end{array}$ & $\begin{array}{c}5 \cdot 10^{-5} \\
1600\end{array}$ & $\begin{array}{r}2.5 \cdot 10^{-} \\
3200\end{array}$ & & $\begin{array}{l}-5 \\
400\end{array}$ & & $\begin{array}{l}0^{-6} \\
000\end{array}$ & & \\
\hline$S=91$ & -0.6092 & -0.5255 & -0.4315 & -0.3081 & -0.2238 & -0.1552 & & 0973 & & 731 & & \\
\hline$S=101$ & -0.2088 & -0.1626 & -0.1242 & -0.0865 & -0.0648 & -0.0494 & & 0343 & & & & \\
\hline$S=111$ & -0.1584 & -0.1264 & -0.0975 & -0.0680 & -0.0497 & -0.0380 & & 0262 & & 195 & & \\
\hline$S=121$ & -0.1459 & -0.1157 & -0.0890 & -0.0618 & -0.0450 & -0.0344 & & 0236 & & 175 & & \\
\hline$S=131$ & -0.1411 & -0.1114 & -0.0855 & -0.0594 & -0.0435 & -0.0332 & & 0227 & & 168 & & 11 \\
\hline
\end{tabular}

KoBoL parameters: $\nu=0.5, \lambda_{+}=9, \lambda_{-}=-8, c=1$.

Option parameters: $K=100, H=90, r=0.072310, T=0.5$.

Algorithm parameters: $d$-space step, $N$ - number of time steps, $S$ - spot price.

use FFT and iFFT, we need to take into account the truncation and step in the dual space, and not only truncation and step in $x$-space. However, as the order of the process increases, the rate of decay of symbols $\phi^{ \pm}(\xi)$ increases as well. Therefore, if the size of the truncated region (measured by $\pi / d$ ) was good enough for processes of order $\nu<1$, the same size will be even better for processes of order $\nu \geq 1$. In our calculations, we keep the size of the truncated region in $\xi$-space, which was good for processes of order $\nu<1$. To control the discretization error in $\xi$-space, we check that as the size of the truncated region decreases two-fold, and the number of points of the grid remains the same, the prices change by $0.01 \%$ or less. Therefore, it remains to discuss the errors of the approximation of the operators on $\mathbb{R}$, before the discretization. This type of error is controlled as follows. We have two essentially different approximate methods: FWH and IWH. However, in our numerical experiments shown in Table 5.10, the relative differences between prices obtained with these two methods, is very small. Hence, we have certain reasons to believe that the results obtained by the fastest method FWH can be used as a benchmark. The results shown in Table 5.8 demonstrate that FWH-method converges very fast: in few seconds, the relative error becomes $0.5 \%$ or less. For processes of order greater than 1, the convergence is even better. The convergence of $\mathrm{CV}$-method is worse: see Table 5.9. Analysis of relative errors of CV-prices shows that the method may be to imprecise near the barrier: even at $10-15 \%$ from the barrier, to obtain option prices with the relative error of order of $0.5 \%, \mathrm{CV}$-method needs hours of CPU-time. The situation at 1\%-3\% from the barrier is much worse: the relative error remains more than $1 \%$ even after dozen hours of CPUtime are spent. In the out-of-the-money region the accuracy of order $1 \%$ can be reached after $30 \mathrm{sec}$. of calculation, but relative error about $0.5 \%$ requires dozens of minutes of CPU-time.

The details of the numerical example are as follows. We consider the NIG model with parameters $\alpha=40, \beta=1.096402897, \delta=1.251720305$. We choose instantaneous interest rate $r=0.05$, hence the drift parameter $\mu \approx 0$. We compute the prices of down-and-out put option with time to expiry $T=1$ year, 
Table 4: Relative difference between down-and-out put prices in KoBoL model computed with FWH-method and IWH-method

A

\begin{tabular}{|l|c|c|c|c|c|c|}
\hline \multirow{2}{*}{ Spot price } & \multicolumn{2}{|c|}{$N=50$} & \multicolumn{2}{c|}{$N=1600$} \\
& FWH & IWH & Relative difference & FWH & IWH & Relative difference \\
$S=91$ & 0.227974 & 0.229114 & $0.50 \%$ & 0.21841 & 0.221462 & $1.40 \%$ \\
$S=101$ & 0.560376 & 0.561387 & $0.18 \%$ & 0.552269 & 0.554932 & $0.48 \%$ \\
$S=111$ & 0.374033 & 0.374595 & $0.15 \%$ & 0.377151 & 0.378602 & $0.38 \%$ \\
$S=121$ & 0.201517 & 0.201802 & $0.14 \%$ & 0.204235 & 0.204961 & $0.36 \%$ \\
$S=131$ & 0.104253 & 0.104392 & $0.13 \%$ & 0.105407 & 0.105748 & $0.32 \%$ \\
\hline CPU-time, sec & 0.01 & 4 & & 0.32 & 23 & \\
\hline
\end{tabular}

B

\begin{tabular}{|l|c|c|c|c|c|c|}
\hline & \multicolumn{3}{|c|}{$N=50$} & \multicolumn{3}{c|}{$N=1600$} \\
Spot price & FWH & IWH & Relative difference & FWH & IWH & Relative difference \\
\hline$S=91$ & 0.238289 & 0.238641 & $0.15 \%$ & 0.228314 & 0.229998 & $0.74 \%$ \\
$S=101$ & 0.569224 & 0.569517 & $0.05 \%$ & 0.561118 & 0.562274 & $0.21 \%$ \\
$S=111$ & 0.378942 & 0.379102 & $0.04 \%$ & 0.382126 & 0.382736 & $0.16 \%$ \\
$S=121$ & 0.203989 & 0.204069 & $0.04 \%$ & 0.206739 & 0.207029 & $0.14 \%$ \\
$S=131$ & 0.105465 & 0.105506 & $0.04 \%$ & 0.10663 & 0.106778 & $0.14 \%$ \\
\hline CPU-time, sec & 0.035 & 7 & & 1 & 49 & \\
\hline
\end{tabular}

C

\begin{tabular}{|l|c|c|c|c|c|c|}
\hline & \multicolumn{3}{|c|}{$N=50$} & \multicolumn{3}{c|}{$N=1600$} \\
Spot price & FWH & IWH & Relative difference & FWH & IWH & Relative difference \\
\hline$S=91$ & 0.242425 & 0.242568 & $0.06 \%$ & 0.232286 & 0.232871 & $0.25 \%$ \\
$S=101$ & 0.572267 & 0.572382 & $0.02 \%$ & 0.564159 & 0.564755 & $0.11 \%$ \\
$S=111$ & 0.380602 & 0.380665 & $0.02 \%$ & 0.383809 & 0.384123 & $0.08 \%$ \\
$S=121$ & 0.204816 & 0.204847 & $0.02 \%$ & 0.207577 & 0.207723 & $0.07 \%$ \\
$S=131$ & 0.10588 & 0.105896 & $0.02 \%$ & 0.107048 & 0.107123 & $0.07 \%$ \\
\hline CPU-time, sec & 0.07 & 14 & & 2 & 105 & \\
\hline
\end{tabular}

D

\begin{tabular}{|l|c|c|c|c|c|c|}
\hline & \multicolumn{3}{|c|}{$N=50$} & \multicolumn{3}{c|}{$N=1600$} \\
Spot price & FWH & IWH & Relative difference & FWH & IWH & Relative difference \\
\hline$S=91$ & 0.244202 & 0.24427 & $0.03 \%$ & 0.233993 & 0.234317 & $0.14 \%$ \\
$S=101$ & 0.573804 & 0.573851 & $0.01 \%$ & 0.565697 & 0.566011 & $0.06 \%$ \\
$S=111$ & 0.381446 & 0.381471 & $0.01 \%$ & 0.384665 & 0.384825 & $0.04 \%$ \\
$S=121$ & 0.205236 & 0.205248 & $0.01 \%$ & 0.208003 & 0.208074 & $0.03 \%$ \\
$S=131$ & 0.106092 & 0.106098 & $0.01 \%$ & 0.107262 & 0.107297 & $0.03 \%$ \\
\hline CPU-time, sec & 0.14 & 31 & & 5 & 237 & \\
\hline
\end{tabular}

E

\begin{tabular}{|l|c|c|c|c|c|c|}
\hline & \multicolumn{2}{|c|}{$N=50$} & \multicolumn{2}{c|}{$N=1600$} \\
Spot price & FWH & IWH & Relative difference & FWH & IWH & Relative difference \\
\hline$S=91$ & 0.245292 & 0.245325 & $0.01 \%$ & 0.23504 & 0.235194 & $0.07 \%$ \\
$S=101$ & 0.574738 & 0.574751 & $0.00 \%$ & 0.56663 & 0.566769 & $0.02 \%$ \\
$S=111$ & 0.381963 & 0.381966 & $0.00 \%$ & 0.385188 & 0.385249 & $0.02 \%$ \\
$S=121$ & 0.205496 & 0.205493 & $0.00 \%$ & 0.208265 & 0.208286 & $0.01 \%$ \\
$S=131$ & 0.106229 & 0.10622 & $-0.01 \%$ & 0.107398 & 0.107401 & $0.00 \%$ \\
\hline CPU-time, sec & 0.35 & 65 & & 12 & 489 & \\
\hline
\end{tabular}

KoBoL parameters: $\nu=0.5, \lambda_{+}=9, \lambda_{-}=-8, c=1$.

Option parameters: $K=100, H=90, r=0.072310, T=0.5$.

Algorithm parameters: $d$-space step, $N$ - number of time steps, $S$ - spot price.

Panel A: $d=0.0025$; Panel B: $d=0.001$; Panel C: $d=0.0005$; Panel D: $d=0.00025$; Panel

$\mathrm{E}: d=0.0001$. 
Table 5: Down-and-out put prices in KoBoL model, $\nu=0.5, T=0.1$

\begin{tabular}{|l|c|c|c|c|c|c|c|}
\hline & ExtFDS & \multicolumn{3}{|c|}{ FWH } & \multicolumn{3}{c|}{ CV } \\
\hline Spot price & & $d=0.00025$ & $d=0.0001$ & ExtFWH & $d=0.000002$ & $d=0.000001$ & ExtCV \\
\hline$S=91$ & 2.349327 & 2.336433 & 2.344786 & 2.349798 & 2.247170 & 2.271554 & 2.291061 \\
$S=101$ & 1.009248 & 1.008639 & 1.009328 & 1.009741 & 1.003694 & 1.005384 & 1.006736 \\
$S=111$ & 0.177806 & 0.177632 & 0.177795 & 0.177893 & 0.176402 & 0.176776 & 0.177075 \\
$S=121$ & 0.049380 & 0.049350 & 0.049381 & 0.049400 & 0.048932 & 0.049045 & 0.049135 \\
$S=131$ & 0.017067 & 0.017051 & 0.017068 & 0.017078 & 0.016900 & 0.016941 & 0.016973 \\
\hline CPU-time(sec) & 99,035 & 3 & 7 & & 18,311 & 96,444 & \\
\hline
\end{tabular}

Relative errors

\begin{tabular}{|l|c|c|c|c|c|c|c|}
\hline & FDS & \multicolumn{3}{|c|}{ FWH } & \multicolumn{3}{c|}{ CV } \\
\hline Spot price & & $d=0.00025$ & $d=0.0001$ & ExtFWH & $d=0.000002$ & $d=0.000001$ & ExtCV \\
\hline$S=91$ & 2.349327 & -0.005488 & -0.001933 & 0.000200 & -0.043483 & -0.033104 & -0.024801 \\
$S=101$ & 1.009248 & -0.000604 & 0.000079 & 0.000488 & -0.005504 & -0.003829 & -0.002490 \\
$S=111$ & 0.177806 & -0.000976 & -0.000060 & 0.000490 & -0.007895 & -0.005790 & -0.004107 \\
$S=121$ & 0.049380 & -0.000612 & 0.000016 & 0.000393 & -0.009082 & -0.006794 & -0.004963 \\
$S=131$ & 0.017067 & -0.000914 & 0.000082 & 0.000680 & -0.009762 & -0.007372 & -0.005461 \\
\hline
\end{tabular}

KoBoL parameters: $\nu=0.5, \lambda_{+}=9, \lambda_{-}=-8, c=1$.

Option parameters: $K=100, H=90, r=0.072310, T=0.1$.

Algorithm parameters: $d$ - space step, $N=400$ - number of time steps, $S-$ spot price.

Table 6: Down-and-out put prices in KoBoL model, FWH-method

\begin{tabular}{|l|c|c|c||c|c|c|}
\hline \multicolumn{2}{l|}{} & \multicolumn{2}{c|}{ Option price, $V_{0.0005, N}(S)$} & \multicolumn{3}{c|}{ Relative errors w.r.t. FDS } \\
\hline Spot price & $N=25$ & $N=50$ & $N=100$ & $N=25$ & $N=50$ & $N=100$ \\
$S=91$ & 2.394927 & 2.356585 & 2.337316 & 0.019410 & 0.003089 & -0.005112 \\
$S=101$ & 0.991945 & 1.000184 & 1.004363 & -0.017145 & -0.008981 & -0.004841 \\
$S=111$ & 0.176471 & 0.176972 & 0.17722 & -0.007506 & -0.004688 & -0.003293 \\
$S=121$ & 0.049410 & 0.049355 & 0.049326 & 0.000603 & -0.000510 & -0.001098 \\
$S=131$ & 0.017114 & 0.017056 & 0.017027 & 0.002777 & -0.000621 & -0.002320 \\
\hline CPU-time(sec) & 0.05 & 0.1 & 0.2 & & & \\
\hline
\end{tabular}

KoBoL parameters: $\nu=0.5, \lambda_{+}=9, \lambda_{-}=-8, c=1$.

Option parameters: $K=100, H=90, r=0.072310, T=0.1$.

Algorithm parameters: $d=5 \cdot 10^{-4}$ - space step, $N$ - number of time steps, $S$ - spot price. 
Table 7: Down-and-out put prices in KoBoL model, CV-method

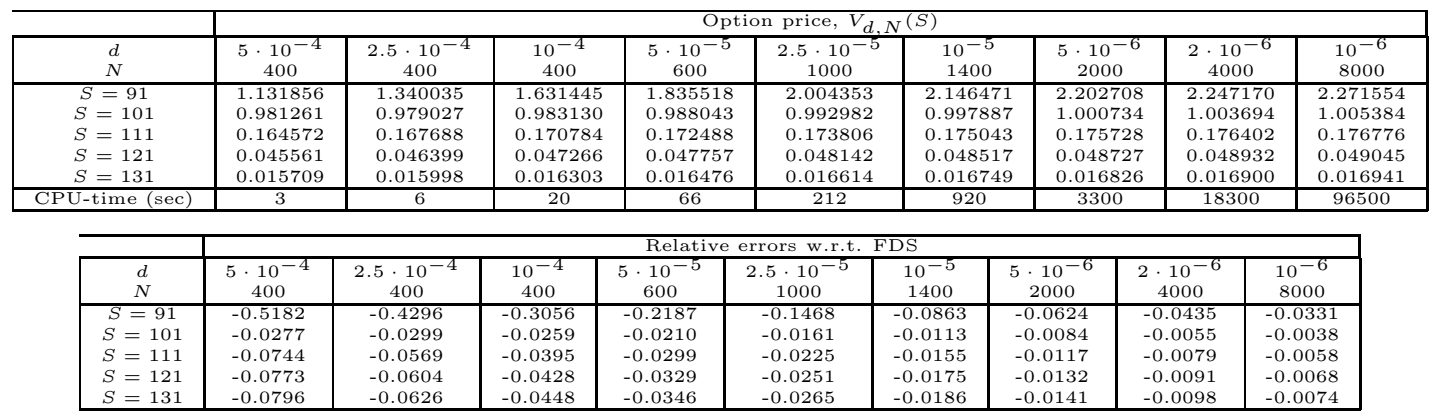

KoBoL parameters: $\nu=0.5, \lambda_{+}=9, \lambda_{-}=-8, c=1$.

Option parameters: $K=100, H=90, r=0.072310, T=0.1$.

Algorithm parameters: $d$-space step, $N$ - number of time steps, $S$ - spot price.

strike price $K=100$ and the barrier $H=80$. First, we choose localization domain $\left(x_{\min } ; x_{\max }\right)$ with $x_{\min }=-\ln 2$ and $x_{\max }=\ln 2$, and calculate the option prices by the FWH method for a fixed space step $(d=0.0025,0.001$, $0.0005, \ldots, 0.00001)$ and number of time steps $(n=50,100,200, \ldots, 1600)$. Second, we increase localization domain two-fold keeping the $x$-step fixed. This implies that the number of points, $M$, will increase by the factor of 2 . Our calculations show that the results remains the same up to the relative error of order $0.01 \%$ or less. Hence, the choice of the localization parameters $x_{\min }=-\ln 2$ and $x_{\max }=\ln 2$ is sufficiently good.

\section{Conclusion}

In the paper, we suggest two new fast and accurate methods, Fast Wiener-Hopf method (FWH-method) and Iterative Wiener-Hopf method (IWH-method), for pricing barrier options for a wide class of Lévy processes. Both methods use the Wiener-Hopf factorization and Fast Fourier Transform algorithm. Using an accurate albeit relatively slow finite-difference algorithm developed in Levendorskii et al (2006) (FDS-method), we demonstrate the accuracy and fast convergence of the two methods for processes of finite variation. We explain that the convergence of the methods must be better for processes of infinite variation, and, as a certain supporting evidence, demonstrate with numerical examples that the results obtained by two methods are in extremely good agreement. Finally, we use FDS, FWH and IWH-methods to demonstrate that Cont-Volchkova method, which is based on the approximation of small jumps by an additional diffusion, lead to sizable relative errors, especially near the barrier and strike.

\section{References}

[1] Albanese, C., and A.Kuznetsov, 2003, "Discretezation schemes for subordinated processes". Working paper 
[2] Amin, K., 1993, "Jump-diffusion option valuation in discrete time", J. Finance, 48, 1833-1863.

[3] Alili, L., and A.Kyprianou, 2005, "Some remarks on first passage of Levy processes, the American put option and pasting principals", Annals of Applied Probability, 15, No 3, 2062-2080.

[4] Avram, F., T. Chan, and M.Usabel, 2002, "On the valuation of constant barrier options under spectrally one-sided exponential Lévy models and Carr's approximation for American puts", Stoch. Proc. Appl., 100, 75-107.

[5] Asmussen, A., F. Avram, and M.R. Pistorius, 2004, "Russian and American put options under exponential phase-type Lévy models", Stoch. Proc. Appl., 109, 79-111.

[6] Barndorff-Nielsen, O. E., 1998, "Processes of Normal Inverse Gaussian Type", Finance and Stochastics, 2, 41-68.

[7] Barndorff-Nielsen, O. E., and S. Levendorskii, 2001, "Feller Processes of Normal Inverse Gaussian type", Quantitative Finance, 1, 318-331.

[8] Boyarchenko, N., and S. Levendorskii,, 2007, "On errors and bias of Fourier transform methods in quadratic term structure models", International Journal of Theoretical and Applied Finance, 10, No. 2, 273-306

[9] Boyarchenko, S. I., and S. Z. Levendorskii, 1999, "Generalizations of the Black-Scholes equation for truncated Lévy processes", Working Paper, University of Pennsylvania, Philadelphia.

[10] Boyarchenko, S. I., and S. Z. Levendorskii, 2000, "Option pricing for truncated Lévy processes", International Journal of Theoretical and Applied Finance, 3, 549-552.

[11] Boyarchenko, S. I., and S. Z. Levendorskii, 2002a, "Perpetual American options under Lévy processes", SIAM Journal of Control and Optimization, 40, 1663-1696.

[12] Boyarchenko, S. I., and S. Z. Levendorskii, 2002b, Non-Gaussian MertonBlack-Scholes theory, World Scientific, New Jercey, London, Singapore, Hong Kong.

[13] Boyarchenko, S. I., and S. Z. Levendorskii, 2002c, "Barrier options and touch-and-out options under regular Levy processes of exponential type", Annals of Applied Probability, 12:4, 1261-1298.

[14] Boyarchenko S.I. and S.Z. Levendorskii (2005): "American options: the EPV pricing model", Annals of Finance, 1:3, 267-292.

[15] Boyarchenko S.I. and S.Z. Levendorskii (2006): "American options in regime-switching models", Working paper, http://ssrn.com/abstract $=929215$

[16] Boyarchenko S.I. and S.Z. Levendorskii (2006): "American options in Lévy models with stochastic interest rates", Working paper, http://ssrn.com/abstract $=1015409$ 
[17] Broadie, M., and J. Detemple, 2004, "Option pricing: valuation models and applications," Management Science 50, 1145-1177

[18] Carr, P., 1998, "Randomization and the American put", Review of Financial Studies, 11, 597-626.

[19] Carr, P., and D. Faguet, 1994, "Fast accurate valuation of American options", Working paper, Cornell University, Ithaca.

[20] Carr, P., H. Geman, D.B. Madan, and M. Yor, 2002, "The fine structure of asset returns: an empirical investigation", Journal of Business, 75, 305-332.

[21] Carr, P., and A. Hirsa, 2003, "Why be backward?", Risk January 2003, 26, 103-107.

[22] Cont, R., and P.Tankov, 2004, Financial modelling with jump processes, Chapman \& Hall/CRC Press.

[23] Cont, R., and E. Voltchkova, 2005, "A finite difference scheme for option pricing in jump diffusion and exponential Lévy models.", SIAM Journal on Numerical Analysis, 43, No. 4, 1596-1626.

[24] Eberlein, E., and U. Keller, 1995, "Hyperbolic distributions in finance", Bernoulli, 1, 281-299.

[25] Eberlein, E., U. Keller and K. Prause, 1998, "New insights into smile, mispricing and value at risk: The hyperbolic model", Journal of Business, 71, 371-406.

[26] Eskin, G.I., 1973, Boundary Problems for Elliptic Pseudo-Differential Equations. Nauka, Moscow (Transl. of Mathematical Monographs 52 Providence. Rhode Island: Amer. Math. Soc. 1980).

[27] Glasserman, P., 2003, Monte Carlo Methods in Financial Engineering, Springer: New York.

[28] Hirsa, A., and D.B. Madan, 2003, "Pricing American options under Variance Gamma", Journal of Computational Finance, 7:2.

[29] Hörmaner, L., 1985, Analysis of Partial Differential Opertors. Vol. III, Springer-Verlag, Berlin Heidelberg New-York.

[30] I. Koponen, 1995, "Analytic approach to the problem of convergence of truncated Lévy flights towards the Gaussian stochastic process", Physics Review E, 52, 1197-1199.

[31] S.G. Kou, 2002, "A jump-diffusion model for option pricing", Management Science, 48, 1086-1101

[32] S. Kou and H. Wang, 2003, "First passage times of a jump diffusion process", Advances in Applied Probability, 35, 504-531.

[33] Kudryavtsev, O.E., and S.Z. Levendorskii, 2006, "Pricing of first touch digitals under normal inverse Gaussian processes, International Journal of Theoretical and Applied Finance, Vol. 9, No. 6, 915-949. 
[34] Kyprianou, A.E., And M.R. Pistorius (2003): "Perpetual options and Canadization through fluctuation theory", Annals of Applied Probability, 13, 1077-1098

[35] Levendorskii, S.Z., 2004a, "Pricing of the American put under Lévy processes", International Journal of Theoretical and Applied Finance, 7, 303335 .

[36] Levendorskii, S.Z., 2004b, "Early exercise boundary and option pricing in Lévy driven models", Quantitative Finance 4 , 525-547

[37] Levendorskii, S.Z., 2005, "Pseudo-diffusions and quadratic term structure models", Mathematical Finance, 15, 393-424.

[38] Levendorskii, S., Kudryavtsev, O., and V.Zherder, 2006 "The relative efficiency of numerical methods for pricing American options under Lévy processes", Journal of Computational Finance, Vol. 9. No 2, Winter 2005/06.

[39] Lipton, A., 2002, “Assets with jumps", Risk (September 2002) 149-153.

[40] Madan, D.B., Carr, P., and E. C. Chang, 1998, "The variance Gamma process and option pricing", European Finance Review, 2, 79-105.

[41] Matache, A.-M., Nitsche,P.-A., and C. Schwab, 2005, "Wavelet Galerkin Pricing of American Options on Lévy Driven Assets", Quantitative Finance, Vol. 5, No 4, 403-424.

[42] Metwally, S. and A. Atiya, 2002, "Using Browning bridge for fast simulation of jump-diffusion processes and barrier options", Journal of Derivatives, 10, 43-54.

[43] Press,W., Flannery,B., Teukolsky, S. and W. Vetterling, 1992, Numerical recipes in C: The Art of Scientific Computing, Cambridge Univ. Press, available at www.nr.com.

[44] Sato, K., 1999, Lévy processes and infinitely divisible distributions, Cambridge University Press, Cambridge.

[45] Sepp, A., 2004, "Analytical Pricing of Double-Barrier Options under a Double-Exponential Jump Diffusion Process: Applications of Laplace Transform", International Journal of Theoretical and Applied Finance, Vol.7, No. 2, 151-175 


\section{Contents}

1 Introduction 3

\begin{tabular}{|lll}
2 & Lévy processes and barrier options & 6
\end{tabular}

2.1 General definitions . . . . . . . . . . . . . . . . . . . . . 6

2.2 Lévv processes and PDO . . . . . . . . . . . . . . . . 7

2.3 Regular Lévv processes of exponential tvpe . . . . . . . . . . 8

2.4 The Wiener-Hopf factorization . . . . . . . . . . . . . 9

2.5 The generalized Black-Scholes equation for barrier options . . . . 10

3 Four methods 12

3.1 Analvtic method of lines or Carr's randomization . . . . . . . . 12

$3.2 \quad$ CV-method . . . . . . . . . . . . . . . . 13

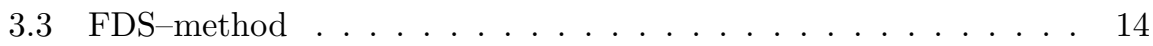

3.4 Iterative Wiener-Hopf method (IWH-method) . . . . . . . . . . 14

3.5 Implicit-explicit Wiener-Hopf method . . . . . . . . . . . . . 16

3.6 IWH-method for more general processes . . . . . . . . . . . . 16

3.7 Fast Wiener-Hopf factorization method (FWH-method) . . . . . 17

4 Approximation of factors in the Wiener-Hopf factorization formula 18

4.1 Realizations of general formulas for $\phi^{ \pm}(\xi)$ using FFT and iFFT . 19

4.2 Approximation of svmbols . . . . . . . . . . . . . . . 20

4.3 Explicit formulas for approximations of $\phi^{ \pm} \ldots \ldots \ldots \ldots 22$

4.4 Approximation of $\phi^{ \pm}(D)$ using Fast Fourier Transform . . . . . . 22

4.5 Algorithm of the FWH-method . . . . . . . . . . . . . 24

5 Numerical Examples $\quad 26$

5.1 Processes of order $\nu<1 \ldots \ldots \ldots \ldots \ldots \ldots$. . . . . . . . 26

5.2 Processes of order $\nu=1$. NIG as an example . . . . . . . . 27

6 Conclusion 31 
Table 8: Down-and-out put prices in NIG model, FWH-method

\begin{tabular}{|c|c|c|c|c|c|c|c|c|c|}
\hline & \multicolumn{9}{|c|}{ Option price, $V_{d, N}(S)$} \\
\hline & & $d=$ & 0025 & & & $d=$ & 0001 & & $d=0$ \\
\hline Spot price & $N=50$ & $N=100$ & $N=200$ & $N=400$ & $N=50$ & $N=100$ & $N=200$ & $N=400$ & $N=800$ \\
\hline$S=81$ & 0.44648 & 0.44012 & 0.43698 & 0.43542 & 0.44871 & 0.44232 & 0.43916 & 0.43759 & 0.43826 \\
\hline$S=91$ & 2.17121 & 2.15012 & 2.13959 & 2.13433 & 2.17345 & 2.15235 & 2.14182 & 2.13656 & 2.13543 \\
\hline$S=101$ & 1.95011 & 1.94701 & 1.94535 & 1.9445 & 1.95137 & 1.94827 & 1.94662 & 1.94577 & 1.94619 \\
\hline$S=111$ & 1.14315 & 1.14964 & 1.15288 & 1.1545 & 1.14367 & 1.15016 & 1.15341 & 1.15503 & 1.15619 \\
\hline$S=121$ & 0.53456 & 0.53933 & 0.54177 & 0.54301 & 0.53474 & 0.53952 & 0.54196 & 0.54319 & 0.54393 \\
\hline CPU-time & 1 & $\frac{2}{2}$ & 4 & 8 & 2 & 5 & 10 & 19 & \\
\hline
\end{tabular}

\begin{tabular}{|c|c|c|c|c|c|c|c|c|}
\hline & \multicolumn{8}{|c|}{ Relative errors w.r.t. $d=0, N=800$} \\
\hline & & $d=$ & 00025 & & & $d=$ & 0001 & \\
\hline Spot price & $N=50$ & $N=100$ & $N=200$ & $N=400$ & $N=50$ & $N=100$ & $N=200$ & $N=400$ \\
\hline$S=81$ & 0.0188 & 0.0043 & -0.0029 & -0.0065 & 0.0239 & 0.0093 & 0.0021 & -0.0015 \\
\hline$S=91$ & 0 & 0.0069 & 0.0019 & & 0.0 & 0.0079 & & 0.0005 \\
\hline$S=101$ & 0.0020 & 0.0004 & -0.0004 & -0.0009 & 0 . & 0.0011 & 02 & -0.0002 \\
\hline$S=111$ & -0.0113 & -0.0057 & -0.0029 & -0.0015 & -0 & -0.0052 & -0.0024 & 010 \\
\hline$S=121$ & -0.0172 & -0.0085 & -0.0040 & -0.0017 & -0.0169 & -0.0081 & -0.0036 & -0.0014 \\
\hline
\end{tabular}

NIG parameters: $\alpha=40, \beta=1.096402897, \delta=1.251720305$.

Option parameters: $K=100, H=80, r=0.05, T=1$.

Algorithm parameters: $d$-space step, $N$ - number of time steps, $S$ - spot price.

$d=0$ denotes the extrapolated prices

Table 9: Down-and-out put prices in NIG model, CV-method

\begin{tabular}{|l|c|c|c|c|c|c|c|c|c|}
\hline \multicolumn{10}{|c|}{ Option price, $V_{d, N}(S)$} \\
$N$ & $10^{-3}$ & $5 \cdot 10^{-4}$ & $2.5 \cdot 10^{-4}$ & $10^{-4}$ & $5 \cdot 10^{-5}$ & $2.5 \cdot 10^{-5}$ & $10^{-5}$ & $5 \cdot 10^{-6}$ & $2 \cdot 10^{-6}$ \\
$N$ & 1,600 & 1,600 & 1,600 & 1,600 & 1,600 & 3,200 & 6,400 & 10,000 & 18,000 \\
\hline$S=81$ & 0.324586 & 0.352655 & 0.378460 & 0.401569 & 0.412402 & 0.419701 & 0.426262 & 0.429568 & 0.432490 \\
$S=91$ & 2.021805 & 2.052380 & 2.075872 & 2.096684 & 2.107450 & 2.114732 & 2.121400 & 2.124734 & 2.127705 \\
$S=101$ & 1.884045 & 1.900767 & 1.913954 & 1.925843 & 1.932057 & 1.935850 & 1.939538 & 1.941296 & 1.942907 \\
$S=111$ & 1.133169 & 1.139239 & 1.144357 & 1.149172 & 1.151754 & 1.153125 & 1.154585 & 1.155233 & 1.155852 \\
$S=121$ & 0.537101 & 0.538817 & 0.540399 & 0.541966 & 0.542834 & 0.543262 & 0.543747 & 0.543953 & 0.544157 \\
\hline CPU-time, sec & 2.5 & 5 & 11 & 29 & 59 & 362 & 2928 & 11370 & 49407 \\
\hline
\end{tabular}

\begin{tabular}{|l|c|c|c|c|c|c|c|c|c|}
\hline & \multicolumn{7}{|c|}{ Relative errors of CV-method w.r.t. FWH-method with $d=0, N=800$} \\
\hline$d$ & $10^{-3}$ & $5 \cdot 10^{-4}$ & $2.5 \cdot 10^{-4}$ & $10^{-4}$ & $5 \cdot 10^{-5}$ & $2.5 \cdot 10^{-5}$ & $10^{-5}$ & $5 \cdot 10^{-6}$ & $2 \cdot 10^{-6}$ \\
$N$ & 1,600 & 1,600 & 1,600 & 1,600 & 1,600 & 3,200 & 6,400 & 10,000 & 18,000 \\
\hline$S=81$ & -0.2594 & -0.1953 & -0.1364 & -0.0837 & -0.0590 & -0.0423 & -0.0274 & -0.0198 & -0.0132 \\
$S=91$ & -0.0532 & -0.0389 & -0.0279 & -0.0181 & -0.0131 & -0.0097 & -0.0066 & -0.0050 & -0.0036 \\
$S=101$ & -0.0319 & -0.0233 & -0.0166 & -0.0105 & -0.0073 & -0.0053 & -0.0034 & -0.0025 & -0.0017 \\
$S=111$ & -0.0199 & -0.0147 & -0.0102 & -0.0061 & -0.0038 & -0.0027 & -0.0014 & -0.0008 & -0.0003 \\
$S=121$ & -0.0126 & -0.0094 & -0.0065 & -0.0036 & -0.0020 & -0.0012 & -0.0003 & 0.0000 & 0.0004 \\
\hline
\end{tabular}

NIG parameters: $\alpha=40, \beta=1.096402897, \delta=1.251720305$.

Option parameters: $K=100, H=80, r=0.05, T=1$.

Algorithm parameters: $d-$ space step, $N-$ number of time steps, $S-$ spot price. 
Table 10: Relative difference between down-and-out put prices in NIG model computed by FWH-method and IWH-method

A

\begin{tabular}{|l|c|c|c|c|c|c|}
\hline \multirow{2}{*}{ Spot price } & \multicolumn{2}{|c|}{$N=100$} & \multicolumn{3}{c|}{$N=1600$} \\
\hline$S=81$ & IWH & FWH & Relative difference & IWH & FWH & Relative difference \\
$S=91$ & 0.40713 & 0.40647 & $-0.16 \%$ & 0.40964 & 0.40103 & $-2.10 \%$ \\
$S=101$ & 2.1184 & 2.11766 & $-0.03 \%$ & 2.10624 & 2.09805 & $-0.39 \%$ \\
$S=111$ & 1.92898 & 1.92854 & $-0.02 \%$ & 1.93000 & 1.92527 & $-0.25 \%$ \\
$S=121$ & 1.14212 & 1.14192 & $-0.02 \%$ & 1.14992 & 1.14792 & $-0.17 \%$ \\
\hline CPU-time, sec & 0.53677 & 0.53670 & $-0.01 \%$ & 0.54197 & 0.54129 & $-0.13 \%$ \\
\hline
\end{tabular}

B

\begin{tabular}{|l|c|c|c|c|c|c|}
\hline & \multicolumn{2}{|c|}{$N=100$} & \multicolumn{3}{c|}{$N=1600$} \\
Spot price & IWH & FWH & Relative difference & IWH & FWH & Relative difference \\
\hline$S=81$ & 0.42941 & 0.42927 & $-0.03 \%$ & 0.42559 & 0.42354 & $-0.48 \%$ \\
$S=91$ & 2.13924 & 2.13906 & $-0.01 \%$ & 2.12151 & 2.11938 & $-0.10 \%$ \\
$S=101$ & 1.94083 & 1.94073 & $-0.01 \%$ & 1.93876 & 1.93754 & $-0.06 \%$ \\
$S=111$ & 1.14707 & 1.14703 & $0.00 \%$ & 1.1536 & 1.15309 & $-0.04 \%$ \\
$S=121$ & 0.53845 & 0.53844 & $0.00 \%$ & 0.54321 & 0.54303 & $-0.03 \%$ \\
\hline CPU-time, sec & 6 & 0.45 & & 53 & 7 & \\
\hline
\end{tabular}

C

\begin{tabular}{|l|c|c|c|c|c|c|}
\hline \multirow{2}{*}{ Spot price } & \multicolumn{2}{|c|}{$N=100$} & \multicolumn{3}{c|}{$N=1600$} \\
$S=81$ & IWH & FWH & Relative difference & IWH & FWH & Relative difference \\
$S=91$ & 0.43639 & 0.43634 & $-0.01 \%$ & 0.43121 & 0.43052 & $-0.16 \%$ \\
$S=101$ & 2.14647 & 2.14642 & $0.00 \%$ & 2.12741 & 2.12671 & $-0.03 \%$ \\
$S=111$ & 1.94494 & 1.94491 & $0.00 \%$ & 1.94214 & 1.94174 & $-0.02 \%$ \\
$S=121$ & 1.14877 & 1.14876 & $0.00 \%$ & 1.155 & 1.15483 & $-0.01 \%$ \\
\hline CPU-time, sec & 0.53904 & 0.53903 & $0.00 \%$ & 0.54369 & 0.54363 & $-0.01 \%$ \\
\hline
\end{tabular}

D

\begin{tabular}{|l|c|c|c|c|c|c|}
\hline \multirow{2}{*}{ Spot price } & \multicolumn{3}{|c|}{$N=100$} & \multicolumn{3}{c|}{$N=1600$} \\
\hline$S=81$ & IWH & FWH & Relative difference & IWH & FWH & Relative difference \\
$S=91$ & 0.44014 & 0.44012 & $0.00 \%$ & 0.43447 & 0.43425 & $-0.05 \%$ \\
$S=101$ & 2.15013 & 2.15012 & $0.00 \%$ & 2.13062 & 2.13039 & $-0.01 \%$ \\
$S=111$ & 1.94702 & 1.94701 & $0.00 \%$ & 1.94398 & 1.94385 & $-0.01 \%$ \\
$S=121$ & 1.14964 & 1.14964 & $0.00 \%$ & 1.15577 & 1.15572 & $0.00 \%$ \\
\hline CPU-time, sec & 0.53934 & 0.53933 & $0.00 \%$ & 0.54396 & 0.54394 & $0.00 \%$ \\
\hline
\end{tabular}

E

\begin{tabular}{|l|c|c|c|c|c|c|}
\hline & \multicolumn{2}{|c|}{$N=100$} & \multicolumn{3}{c|}{$N=1600$} \\
Spot price & IWH & FWH & Relative difference & IWH & FWH & Relative difference \\
\hline$S=81$ & 0.44232 & 0.44232 & $0.00 \%$ & 0.43647 & 0.43642 & $-0.01 \%$ \\
$S=91$ & 2.15236 & 2.15235 & $0.00 \%$ & 2.13268 & 2.13262 & $0.00 \%$ \\
$S=101$ & 1.94828 & 1.94827 & $0.00 \%$ & 1.94516 & 1.94513 & $0.00 \%$ \\
$S=111$ & 1.15017 & 1.15016 & $0.00 \%$ & 1.15626 & 1.15625 & $0.00 \%$ \\
$S=121$ & 0.53953 & 0.53952 & $0.00 \%$ & 0.54413 & 0.54412 & $0.00 \%$ \\
\hline CPU-time, sec & 67 & 5 & & 590 & 77 & \\
\hline
\end{tabular}

NIG parameters: $\alpha=40, \beta=1.096402897, \delta=1.251720305$.

Option parameters: $K=100, H=80, r=0.05, T=1$.

Algorithm parameters: $d$-space step, $N$ - number of time steps, $S$ - spot price.

Panel A: $d=0.0025$; Panel B: $d=0.001$; Panel C: $d=0.0005$; Panel D: $d=0.00025$; Panel

$\mathrm{E}: d=0.0001$. 
Centre de recherche INRIA Paris - Rocquencourt Domaine de Voluceau - Rocquencourt - BP 105 - 78153 Le Chesnay Cedex (France)

Centre de recherche INRIA Bordeaux - Sud Ouest : Domaine Universitaire - 351, cours de la Libération - 33405 Talence Cedex Centre de recherche INRIA Grenoble - Rhône-Alpes : 655, avenue de l'Europe - 38334 Montbonnot Saint-Ismier

Centre de recherche INRIA Lille - Nord Europe : Parc Scientifique de la Haute Borne - 40, avenue Halley - 59650 Villeneuve d'Ascq Centre de recherche INRIA Nancy - Grand Est : LORIA, Technopôle de Nancy-Brabois - Campus scientifique 615, rue du Jardin Botanique - BP 101 - 54602 Villers-lès-Nancy Cedex

Centre de recherche INRIA Rennes - Bretagne Atlantique : IRISA, Campus universitaire de Beaulieu - 35042 Rennes Cedex Centre de recherche INRIA Saclay - Île-de-France : Parc Orsay Université - ZAC des Vignes : 4, rue Jacques Monod - 91893 Orsay Cedex

Centre de recherche INRIA Sophia Antipolis - Méditerranée : 2004, route des Lucioles - BP 93 - 06902 Sophia Antipolis Cedex 